\title{
Orhaneli İlçesinin Vasküler Bitki Çeşitliliği (Bursa, Türkiye)
}

\author{
Gülşah BAĞÇIVAN ${ }^{\mathscr{\&}}$, Ruziye DAŞKIN² \\ ${ }^{1}$ Bursa Uludağ University, Büyükorhan Vocational High School, Department of Forestry, 16990, Büyükorhan, Bursa, ${ }^{2}$ Bursa Uludag \\ University, Faculty of Arts and Sciences, Department of Biology, 16059, Görükle, Bursa, Turkey \\ ${ }^{1}$ https://orcid.org/0000-0001-6131-8811, 2https://orcid.org/0000-0003-3258-5595 \\ $\bowtie$ : glshbagcivan@uludag.edu.tr
}

\section{ÖZET}

$\mathrm{Bu}$ çalışma Bursa ilinin güneyinde bulunan Orhaneli ilçesindeki vasküler bitki çeşitliliğini kapsamaktadır. Çalışma alanından 47 farklı istasyondan 2100 bitki örneği toplanmış ve bu örneklerin teşhisi sonunda 82 familya 347 cinse ait 713 takson (485 tür, 157 alt tür, 70 varyete ve 1 melez) tespit edilmiştir. $\mathrm{Bu}$ taksonlardan 14'ü Pteridophyta bölümüne, 699'u Spermatophyta bölümüne ait olduğu belirlenmiştir. 699 taksondan 8'i Pinophytina, 691’i Magnoliophytina alt bölümündedir. 691 taksondan 583'ü Magnoliopsida, 108'i Liliopsida sınıfındadır. Bitkilerin fitocoğrafik bölgelere göre dağılımları ve oranları Akdeniz elementi 149 (\% 20.9), Avrupa-Sibirya elementi 89 (\% 12.5), İran-Turan elementi 50 (\%7) ve çok bölgeli yada bölgesi bilinmeyenler 425 (\% 59.6) şeklindedir. Endemik taksonların tehlike kategorileri de değerlendirilmiştir. Taksonların Raunkier'in hayat formlarına göre dağılımları ve oranları 59 (\% 8.3)'u Fanerofit, 328 (\% 46)'i Hemikriptofit, 78 (\% 10.9)'i Kriptofit, 231 (\% 32.4)'i Terofit, 13 (\%1.8)'ü Kamefit ve 4 (\% 0.6)'ü Epifit olarak bulunmuştur. En fazla takson içeren familyalar ve takson sayıları sırasıyla, Asteraceae (83), Fabaceae (64), Poaceae (50), Lamiaceae (45), Brassicaceae (42) ve Caryophyllaceae (41)'dir. En fazla takson içeren cinsler ve takson sayıları ise Trifolium L. (18), Alyssum L., Galium L., Verbascum L. (12), Vicia L., Hypericum L. (11), Euphorbia L. (9) olarak bulunmuştur.

\section{Vascular Plant Diversity of Orhaneli District (Bursa, Turkey)}
ABSTRACT
This study consists of vascular plants of Orhaneli district that is located in the south of Bursa province. During the study, 2100 plant specimens were collected from 47 different stations in the research area. At the end of identification, 713 taxa (485 species, 157 subspecies, 70 varieties and 1 hybrid) belonging to 347 genera and 82 families were determined. The distribution of total taxa according to divisio, subdivisio and classis is as follows: 14 taxa in Pteridophyta division, 699 taxa in Spermatophyta division, 8 taxa in Pinophytina subdivision, 691 taxa in Magnoliophytina subdivision, 583 taxa in Magnoliopsida and 108 taxa in Liliopsida classis. The distribution of the taxa according to the phytogeographical regions and their rates are as follows; Mediterranean element 149 (20.9\%), Euro-Siberian element 89 (12.5\%), Irano-Turanian element $50(7 \%)$ and multiregional or unknown originated 425 (59.6\%). The threat categories of 57 endemic taxa are presented. The threat categories of these endemic taxa are presented. The largest families are respectively, Asteraceae (83), Fabaceae (64), Poaceae (50), Lamiaceae (45), Brassicaceae (42) and Caryophyllaceae (41). The genera containing highest number of taxa are Trifolium L. (18), Alyssum L., Galium L., Verbascum L. (12), Vicia L., Hypericum L. (11), Euphorbia L. (9).

Araştırma Makalesi

Makale Tarihçesi
Geliş Tarihi $\quad: 04.10 .2019$
Kabul Tarihi $\quad: 19.12 .2019$

Anahtar Kelimeler
Vasküler bitkiler
Flora
Orhaneli
Bursa
Türkiye

\section{Research Article}

$\begin{array}{ll}\text { Article History } & \\ \text { Received } & : 04.10 .2019 \\ \text { Accepted } & : 19.12 .2019\end{array}$

Keywords
Vascular plants
Flora
Orhaneli
Bursa
Turkey

To Cite : Bağçıvan G, Daşkın R 2020. Orhaneli İlçesinin Vasküler Bitki Çeşitliliği (Bursa, Türkiye). KSU Tarım ve Doğa Derg 23 (2): 416-434. DOI: 10.18016/ksutarimdoga.vi.629511 


\section{GİRIŞ}

Türkiye, bitkisel çeşitlilik açısından kuzey yarımküredeki önemli alanlardan birisidir. Türkiye Florası 11.707 taksondan oluşmaktadır. Bu taksonlardan 3649'u Türkiye'ye özgü olup endemizm oranı yaklaşık \%32'dir (Güner ve ark., 2012). Endemizm oranı diğer Avrupa ülkelerine kıyasla oldukça yüksektir ki bu oran İspanya'da \%18.6, Yunanistan'da \%14.9 ve Fransa'da \%2.9'dur (Avcl, 2005). Endemizm oranının yüksek oluşunun sebepleri arasında, ülkemizin Asya ve Avrupa kitalarının birleştiği yerde, Akdeniz, Avrupa-Sibirya ve İranTuran fitocoğrafik bölgelerinin kesiştiği bir bölgede bulunması, topoğrafyasının kısa mesafelerde değişmesi, eşsiz jeolojik yapısı, çeşitli ana kaya ve toprak tiplerini birlikte bulundurması, farklı iklim tiplerinin etkisi altında oluşu ve jeolojik devirlerde geçirdiği iklimsel değişimleri sayabiliriz.

Bugüne kadar çalışma alanının yakın çevresinde ve Bursa ilinde birçok floristik çalışma yapılmıştır (Çırpıcı, 1989; Dirmenci, 2006; Günay Daşkın ve
Kaynak, 2006; Daşkın ve Kaynak, 2010a,b; Erdoğan ve ark., 2011; Güner ve Akçiçek, 2013; Bağçıvan ve Daşkın, 2019)

$\mathrm{Bu}$ çalışma ile sahip olduğu farklı ana kaya yapısı, zengin yer altı kaynakları ve bitkisel çeşitliliği ile Bursa ilinin dikkat çeken ilçelerinden Orhaneli'de yetişen vasküler bitkilerin belirlenmesi bu sayede Bursa ili ve Türkiye Florasına katkı sağlanması amaçlanmıştır.

\section{MATERYAL ve METOT \\ Çalışma Alanının Özellikleri}

Bursa ilinin güneyinde Uludağ'ın eteklerinde yer alan Orhaneli ilçesi yaklaşık $764 \mathrm{~km}^{2}$ olup yüksekliği 487 m'dir. Kuzeyinde Nilüfer ve Osmangazi, güneyinde Büyükorhan, Harmancik, batısında Mustafakemalpaşa, doğusunda Keles ilçeleriyle çevrilidir. Türkiye Florası'nda kullanılan kareleme sistemine göre (Davis, 1965), alanın büyük kısmı B2 karesinde yer almaktadır (Şekil 1).
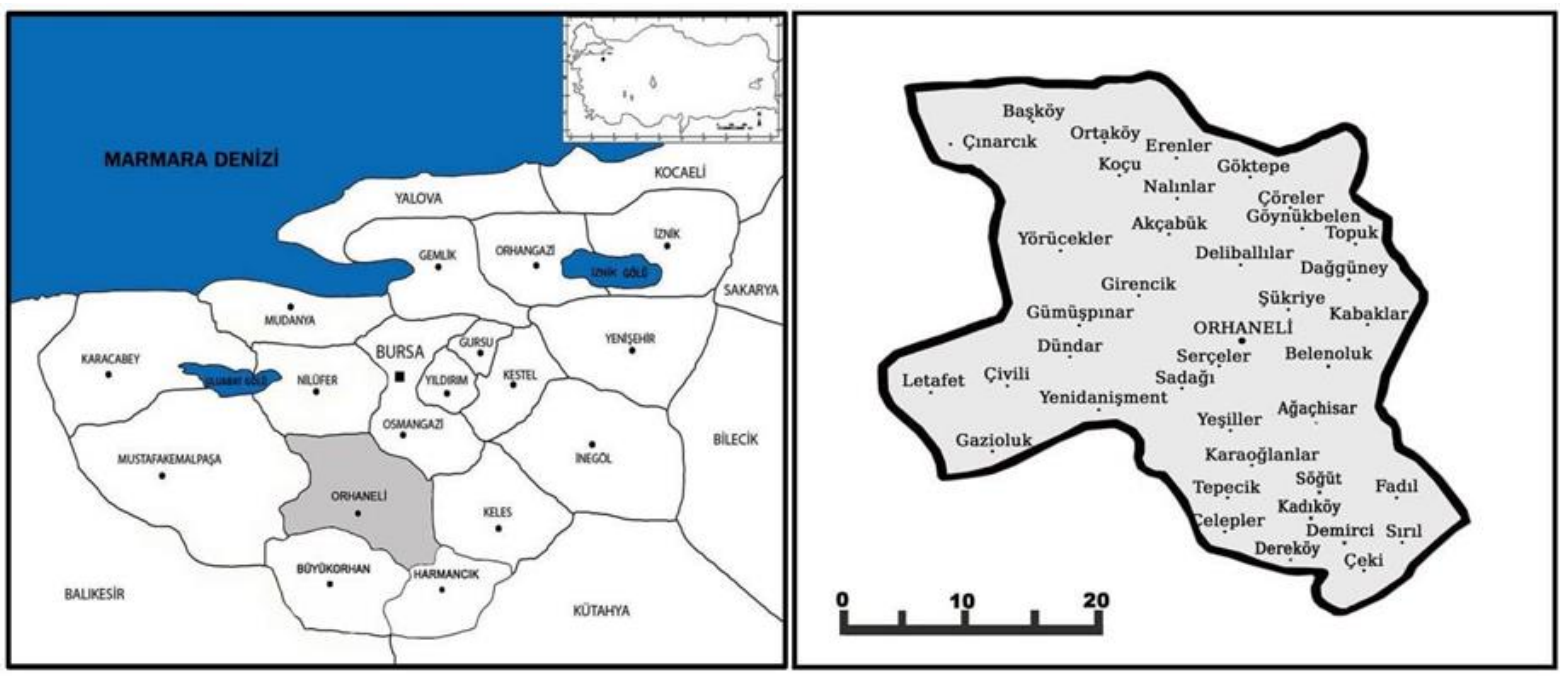

Şekil 1. Çalışma Alanının Haritası

Figure 1. Map of study area

T.C. Çevre ve Orman Bakanlığı, Devlet Meteoroloji İşleri Genel Müdürlüğü ve Bursa Meteoroloji İl Müdürlügünden alınan meteorolojik verilere göre Bursa'da yıllık ortalama sıcaklık $14.5{ }^{\circ} \mathrm{C}$, yıllık ortalama yağış 56.4 mm (1976-2006, 31 yıl) Orhaneli'de yıllık ortalama sıcaklık $13.6^{\circ} \mathrm{C}$, yıllık ortalama yağış $44.6 \mathrm{~mm}$ (2013-2016, 4 yıl) olarak ölçülmüştür (Anonim, 1976 2006; Anonim, 2013-2016). Alanın iklimi Akdeniz olup yarı kurak Akdeniz biyoiklim tipinin kışları soğuk alt tipine girmektedir. İklimsel veriler Emberger metoduna göre hesaplanmıştır (Akman, 1999).

Çalışma alanında Pinus nigra J. F. Arnold subsp. nigra var. caramanica (Loudon) Rehd., P. brutia Ten.'nın karışık halde bulunduğu kurak ormanlar hakimdir. Çam ormanlarının yangın, kesim gibi nedenlerle tahrip olduğu yerlerde Juniperus oxycedrus L. ssp. oxycedrus, Phyllirea latifolia L., Paliurus spinachristii Mill., Quercus infectoria Oliv. ssp. infectoria, Rhus coriaria L., Pistacia palaestina Boiss., Cistus creticus L., C. salviifolius L., Arbutus andrachne L. türlerinden oluşan çalı vejetasyonu görülmektedir. Nemli vadi tabanı ve içlerinde Fagus orientalis Lipsky, Alnus glutinosa (L.) Gaertn. subsp. glutinosa,
Platanus orientalis L., Populus tremula L. subsp. tremula, Tilia tomentosa Moench, Corylus avellana L. var. avellana yaygin olup akarsu ve su kanalı kenarlarında Phragmites australis (Cav.) Trin. ex Steud., Vitex agnus castus L., Mentha longifolia L. subsp. thyphoides (Briq.) Harley, M. pulegium L., Rumex crispus L., Polygonum persicaria L., Epilobium hirsutum L., Lythrum salicaria L., Urtica dioica L., Plantago major L. subsp. intermedia (Gilib.) Lange ile Salix L., Cyperus L. ve Juncus L. türleri yetişmektedir. Alanda Taxus baccata L. sadece Sadağı Kanyonu içlerinde lokal olarak bulunmaktadır. 
Çalışma alanı jeolojik olarak esasen magmatik kayalardan oluşmaktadır. Erenler mevkii'nden başlayarak Harmancık ilçesine kadar anakaya olarak devam eden ofiyolit seri yer yer bazı yerlerde aşınma ile serpantin kayalar veya taşlık alanlar şeklinde görülmektedir. Alan linyit, mermer, granit, krom, nikel ve olivin madeni sicak su, maden suyu gibi yeraltı kaynaklarına da sahiptir. Gümüşpınar ve Dündar çevresinden çıkarılan linyit kömürü, Orhaneli Termik Santralinde enerjiye dönüştürülmekte, krom, nikel ve olivin madeni ise ŞETAT Madenciliğin tesislerinde işlenmektedir. Ayrıca ilçede çeşitli şirketlere ait mermer ve granit ocakları da faaliyet göstermektedir (Anonim, 2010; 2012). Sadağı Köyü üstlerindeki Sadağı Kanyonundaki Kaya Hamamları ve Ağaçhisar Köyündeki kaplıcalar termal turizm açısından önemli bir potansiyele sahiptir. Sadağı Kanyonundaki eski hamam kalıntılarının bulunduğu yerdeki kaynaktan çıkan sıcaksu borularla vadi boyunca taşınarak Serçeler köyü civarındaki Aslanlı Jeotermal tesislerinde kullanılmaktadır.

Sadağı Kanyonu eşsiz doğal güzellikleri, sahip olduğu jeolojik yapısı, su kaynakları ve bitki çeşitliliği açısından alanın en dikkat çekici yerlerindendir. $\mathrm{Bu}$ özelliklerinden dolayı her yıl çok sayıda yerli ve yabanc1 turist tarafindan ziyaret edilmekte olup Orman ve Su İşleri Bakanlığı tarafindan 436 ha'lık alanı Tabiat Parkı olarak ilan edilmiştir.

\section{Bitki Örneklerinin Toplanması, Teşhisi ve Listelenmesi}

$\mathrm{Bu}$ çalışmanın materyalini oluşturan yaklaşık 2100 bitki örneği, 2011-2017 yılları arasında farklı vejetasyon periyodlarında alandaki 47 lokaliteden toplanmıştır. Çalışma sırasında toplanan örnekler herbaryum materyali haline getirilerek Bursa Uludağ Üniversitesi Herbaryumunda (BULU) saklanmaktadır. Bitki örneklerinin teşhisinde başta Türkiye Florası (Davis, 1965-1985; Davis ve ark., 1988; Güner ve ark., 2000) olmak üzere bazı resimli kitaplardan (Seçmen ve Leblebici, 2008; Malyer ve ark., 2016) yararlanılmıştır. Bitkilerin adları ve statü değişiklikleri Türkiye Bitkileri listesi (Damarlı Bitkiler) (Güner ve ark., 2012) isimli kitaptan kontrol edilmiştir. Hayat formları belirlenirken Raunkier (1934)'den yararlanılmıştır. Bitki listesinde sırasıyla eğreltiler, gymnospermler ve angiospermler verilmiştir. $\mathrm{Bu}$ bölüm ve alt bölümlerde yer alan familya, cins ve tür isimleri kendi içlerinde alfabetik olarak listelenmiştir. Bitki listesi verilirken bitkinin latince ismi, yazarı, lokalitesi, toplanma tarihi, fitocoğrafik bölge, hayat formu, endemizm durumu ve tehlike kategorisi verilmiştir.

Simgeler ve Kısaltmalar; Akd.: Akdeniz Elementi, Avr.-Sib.: Avrupa-Sibirya Elementi, D. Akd.: Doğu Akdeniz Elementi, CR: Critically Endangered; EN:
Endangered, END.: ENDEMIK, Epi.: Epifit, Fan.: Fanerofit, GB: Gülşah BAĞÇIVAN, Hem.: Hemikriptofit, İr.-Tur.: İran-Turan Elementi, J: Juniperus, KD: Kürşad DAŞKIN, Kam.: Kamefit, Kozm.: Kozmopolit, Kri.: Kriptofit, LC: Least Concern, NE: Not Evaluated, NT: Near Threatened, P: Pinus, RD: Ruziye DAŞKIN, Q.: Quercus, subsp.: Alt tür, Ter.: Terofit, var.: Varyete, VU: Vulnerable, ZB: Ziya BAĞÇIVAN.

Araştırma Alanında Bitki Toplanan Lokaliteler

1. A2(A) Bursa: Orhaneli: Başköy-Çınarcık arası, Cinarcik'a $2 \mathrm{~km}$ kala, RD, GB.

2. A2(A) Bursa: Orhaneli: Başköy çıkışı, 35T 657369 D, $4435736 \mathrm{~K}, 700 \mathrm{~m}, \mathrm{RD}$.

3. A2(A) Bursa: Orhaneli: Çınarcık Barajı üstleri ve çevresi, 35T $650977 \mathrm{D}, 4432753 \mathrm{~K}, 446 \mathrm{~m}, \mathrm{RD}, \mathrm{GB}$.

4. A2(A) Bursa: Orhaneli: Çınarcık Korubaşı köyü arasi, 35T $650183 \mathrm{D}, 4438441 \mathrm{~K}, 430 \mathrm{~m}, \mathrm{RD}$.

5. A2(A) Bursa: Orhaneli; Orhaneli yolu, Göktepe yol ayrımına $500 \mathrm{~m}$ kala, 35T $664493 \mathrm{D}, 4437429 \mathrm{~K}, 626$ $\mathrm{m}, \mathrm{RD}, \mathrm{GB}$.

6. A2(A) Bursa: Orhaneli: Erenler'e $2 \mathrm{~km}$ kala, 35T $662970 \mathrm{D}, 4435035 \mathrm{~K}, 631 \mathrm{~m}, \mathrm{RD}$, GB.

7. B2 Bursa: Orhaneli; Orhaneli Bursa, Erenler'e 1 kala, 35S 661822 D, 4433746 K, 543-630 m, RD, GB, ZB.

8. B2 Bursa: Orhaneli; Nalınlar yol ayrımından $2 \mathrm{~km}$ önce, $495 \mathrm{~m}, \mathrm{RD}$, GB.

9. B2 Bursa: Orhaneli: Yörücekler yol ayrımından 2 km sonra, 35S 6633228 D, $4424277 \mathrm{~K}, 392 \mathrm{~m}, \mathrm{RD}, \mathrm{GB}$. 10. B2 Bursa: Orhaneli; Akçabük köyü Fatih Sakin mesire alanı, 35S 6645090 D, $4424010 \mathrm{~K}, 385 \mathrm{~m}$, GB.

11. B2 Bursa: Orhaneli: Çöreler yol ayrımı çevresi, $35 \mathrm{~S}$ $665742 \mathrm{D}, 4423203 \mathrm{~K}, 355 \mathrm{~m}, \mathrm{RD}$, GB.

12. B2 Bursa: Orhaneli; Orhaneli Deliballılar yol ayrımı arası, 35S $670235 \mathrm{D}, 4414759 \mathrm{~K}, 688 \mathrm{~m}, \mathrm{RD}$, GB.

13. B2 Bursa: Orhaneli; Topuk yol ayrımı Topuk arası, 4. km'si, 35S 671018 D, $4427911 \mathrm{~K}, 536 \mathrm{~m}$, RD, GB.

14. B2 Bursa: Orhaneli; Topuk Göynükbelen yolu, Göynükbelen'e 4 km kala, 35S 672598 D, $4426943 \mathrm{~K}$, $705 \mathrm{~m}, \mathrm{RD}, \mathrm{GB}, \mathrm{ZB}$.

15. B2 Bursa: Orhaneli; Şükriye-Belenoluk yolu üzeri, 35S 672735 D, $4418645 \mathrm{~K}, 672 \mathrm{~m}, \mathrm{RD}$, GB.

16. B2 Bursa: Orhaneli; Kabaklar köyü çıkışı, $39^{\circ}$ 53 '30”K, $29^{\circ} 05^{\prime} 13$ ” D, $578 \mathrm{~m}, \mathrm{RD}$, GB.

17. B2 Bursa: Orhaneli; Ağaçhisar köyüne $5 \mathrm{~km}$ kala, RD, GB.

18. B2 Bursa: Orhaneli; Ağaçhisar Fadıl arası, $3 . \mathrm{km}$, $\mathrm{RD}, \mathrm{GB}$.

19. B2 Bursa: Orhaneli; Fadıl Ağaçhisar arası, Ağaçhisar'a 5 km kala, 35S 679420 D, 4411608 K, 727 $\mathrm{m}, \mathrm{RD}, \mathrm{GB}, \mathrm{ZB}$.

20. B2 Bursa: Orhaneli; Harmancık Orhaneli arası, Orhaneli'ye $4 \mathrm{~km}$ kala, RD, KD.

21. B2 Bursa: Orhaneli; Orhaneli girişi, Gürgür Çeşmesi çevresi, 35S 669256 D, 4420645 K, 417 m, RD, 
$\mathrm{GB}, \mathrm{ZB}$.

22. B2 Bursa: Orhaneli; Orhaneli çıkışı Büyükorhan yolu, $5 \mathrm{~km}, \mathrm{RD}, \mathrm{GB}, \mathrm{ZB}$.

23. B2 Bursa: Orhaneli; Serçeler Orhaneli yolu arası, $447 \mathrm{~m}, \mathrm{RD}, \mathrm{GB}, \mathrm{ZB}$.

24. B2 Bursa: Orhaneli; Sadağı köyü çevresi, 35S $663708 \mathrm{D}, 4416341 \mathrm{~K}, 626 \mathrm{~m}, \mathrm{RD}, \mathrm{KD}$.

25. B2 Bursa: Orhaneli; Serçeler girişi, Aslanlı Jeotermal Tesisleri çevresi, 35S 666137 D, 4417386 K, $441 \mathrm{~m}, \mathrm{RD}, \mathrm{GB}, \mathrm{ZB}$.

26. B2 Bursa: Orhaneli: Sadağı Kanyonu Tabiat Parkı, 35S 663124 D, 4415244 K, 553 m, RD, GB.

27. B2 Bursa: Orhaneli; Yeşiller köyü çıkışı, Sadağı kanyonu üstleri, 35S 662844 D, $4414697 \mathrm{~K}, 772$ m, RD, GB, ZB.

28. B2 Bursa: Orhaneli; Çivili Bal üretim Ormanı çevresi, 35S 651910D, 4416289 K, $641 \mathrm{~m}, \mathrm{RD}$, GB.

29. B2 Bursa: Orhaneli; Çivili Letafet arası, 35S $650470 \mathrm{D}, 4416179 \mathrm{~K}, 807 \mathrm{~m}, \mathrm{RD}, \mathrm{GB}$.

30. B2 Bursa: Orhaneli; Yenidanışment Çivili arası, 1. $\mathrm{km}, 35 \mathrm{~S} 665284 \mathrm{D}, 4416545 \mathrm{~K}, 534 \mathrm{~m}, \mathrm{RD}, \mathrm{GB}$.

31. B2 Bursa: Orhaneli; Gümüşpınar Dündar arası, 35S 658347 D, 4417988 K, 617 m, RD, GB.

32. B2 Bursa: Orhaneli; Dündar Gümüsspinar arası, Gümüşpınar yol ayrımı çevresi, 35S 659972 D, $4418592 \mathrm{~K}, 704 \mathrm{~m}, \mathrm{RD}, \mathrm{GB}, \mathrm{ZB}$.

33. B2 Bursa: Orhaneli; Girencik Gümüşpınar, Gümüşpınar’a $3 \mathrm{~km}$ kala, 35S $660442 \mathrm{D}, 4422201 \mathrm{~K}$, $493 \mathrm{~m}, \mathrm{RD}, \mathrm{GB}$.

34. B2 Bursa: Orhaneli; Gazioluk Aktaş arası, 2. km, 35S $652657 \mathrm{D}, 4412527 \mathrm{~K}, 777 \mathrm{~m}, \mathrm{RD}$, GB.

35. B2 Bursa: Orhaneli; M. Akalan köyü yol ayrımına $1 \mathrm{~km}$ kala, 35S 670300 D, $441644 \mathrm{~K}, 646 \mathrm{~m}$, RD, GB, ZB.

36. B2 Bursa: Orhaneli; Tepecik Özlüce arası, Özlüce girişi, 35S $665453 \mathrm{D}, 4408821 \mathrm{~K}, 744-877 \mathrm{~m}, \mathrm{RD}$, GB.

37. B2 Bursa: Orhaneli; Eskidanışment köyü çıkışı, $39^{\circ} 49^{\prime} 23^{\prime \prime K}, 29^{\circ} 44^{\prime} 29^{\prime \prime} \mathrm{D}, 962 \mathrm{~m}, \mathrm{RD}, \mathrm{GB}$.

38. B2 Bursa: Orhaneli; Söğüt köyü üstleri, $35 \mathrm{~S}$ 674484 D, $4410329 \mathrm{~K}, 1059$ m, RD, GB.

39. B2 Bursa: Orhaneli; Söğüt Dinlenme Tesisleri çevresi, 35S $671582 \mathrm{D}, 4408637 \mathrm{~K}, 856 \mathrm{~m}$, RD, GB.

40. B2 Bursa: Orhaneli; Demirci köyü çevresi, 35S 672117 D, $4407808 \mathrm{~K}, 887 \mathrm{~m}, \mathrm{RD}, \mathrm{GB}, \mathrm{ZB}$.

41. B2 Bursa: Orhaneli; Kadı köyü girişi, 810 m, RD, $\mathrm{KD}$

42. B2 Bursa: Orhaneli; Kadıköy Dereköy yol ayrımı arası, 35S 6670519 D, 4407023 K, 811 m, RD, GB, ZB. 43. B2 Bursa: Orhaneli; Celaplar yol ayrımına $2 \mathrm{~km}$ kala, RD, GB.

44. B2 Bursa: Orhaneli; Harmancık'a 16 km kala, Çeki Sırıl yol ayrımı çevresi, 35S 675482 D, 4404612 K, 965 $\mathrm{m}, \mathrm{RD}, \mathrm{GB}$.

45. B2 Bursa: Orhaneli; Harmancık yolu, Harmancık'a $14 \mathrm{~km}$ kala, 35S 676896 D, 4401689 K, 972 m, RD, GB. 46. B2 Bursa: Orhaneli; Dağgüney girişi, GB, ZB.

47. B2 Bursa: Orhaneli; Karaoğlanlar köyü içi, 760 m, RD, GB.
BULGULAR

Çalışma Alanının Floristik Listesi

Divisio: Pteridophyta (Eğreltiler)

1.ANTHYRIACEAE

Cystopteris fragilis (L.) Bernh., 26, 12.03.2015, BULU 38221, Kri.

2.ASPLENIACEAE

Asplenium adiantum-nigrum L., 8, 17.04.2015, BULU 35499, Kri.

Asplenium ceterach L., 29, 16.04.2014, BULU 35442, Kri. Asplenium ruta-muraria L., 26, 12.03.2015, BULU 38082, Kri.

Asplenium onopteris L. 30, 16.04.2014, BULU 35411, Kri. Asplenium trichomanes L. subsp. trichomanes, 35, 23.06.2017, BULU 41599, Kri.

3.DRYOPTERIDACEAE

Dryopteris filix mas (L.) Schott., 27, 12.03.2015, BULU 38260, Kri.

Dryopteris pallida (Bory) Fomin subsp. pallida, 26, 22.04.2014, BULU 35668, Kri.

\section{EQUISETACEAE}

Equisetum arvense L., 21, 30.07.2017, BULU 42040, Kri. 5.POLYPODIACEAE

Polypodium vulgare L. var. vulgare, 26, 22.04.2014, BULU 35666, Kri.

Polypodium interjectum Shivas, 26, 12.03.2015, BULU 38081, Kri

\section{PTERIDACEAE}

Cheilanthes marantae (L.) R. Br., 6, 11.05.2016, BULU 39585, Kri.

Cheilanthes persica (Bory) Mett. ex Kuhn, 27, 07.04.2016, BULU 39526, Kri.

Pteridium aquilinum (L.) Kuhn, 37, 24.05.2014, BULU 36479, Kri.

Bölüm: Spermatophyta

Alt bölüm: Pinophytina (Açılk Tohumlular)

7.CUPRESSACEAE

Juniperus excelsa M. Bieb. subsp. excelsa, 19, 30.07.2017, BULU 41968, Fan.

Juniperus foetidissima Willd., 26, 22.04.2014, BULU 35672, Fan.

Juniperus oxycedrus L. subsp. oxycedrus, 15, 30.07.2017, BULU 41989, Fan

\section{EPHEDRACEAE}

Ephedra major Host, 35, 23.06.2017, BULU 41562, Fan. Ephedra campylopoda C. A. Mey., 26, 22.04.2014, BULU 35673, Fan.

\section{PINACEAE}

Pinus brutia Ten., 3, 10.06.2014, BULU 37083, Fan.

Pinus nigra J. F. Arnold subsp. nigra var. caramanica (Loudon) Rehd., 9, 17.04.2014, BULU 35535A, Fan.

10.TAXACEAE

Taxus baccata L., 26, 22.04.2014, BULU 35670, Fan.

Alt bölüm: Magnoliophytina (Tohumlu Bitkiler)

Sinff: Liliopsida (Monokotiller)

11.ALISMATACEAE

Alisma plantago-aquatica L. subsp. plantago-aquatica, 26, 13.07.2016, BULU 40230, Avr.-Sib., Hem.

\section{AMARYLLIDACEAE}

Allium ampeloprassum L., 26, 25.06.2015, BULU 38386, Akd., Kri. 
Allium jubatum Macbride, 21, 16.05.2017, BULU 41231, İr.-Tur., Kri.

Allium lycaonicum Siehe ex Hayek, 44, 22.05.2015, BULU 38297, Kri.

Allium paniculatum L. subsp. paniculatum, 26, 25.06.2015, BULU 38379, Akd., Kri.

Allium scorodoprassum L. subsp. rotundum (L.) Stearn, 31, 11.06.2014, BULU 37110, Kri.

Allium sibthorpianum Schult. \& Schult. f., 11, 25.05.2016, BULU 39725, D. Akd., Kri., END., LC.

Galanthus elwesii Hook. f. var. elwesii, 26, 01.03.2016, BULU 39502, Kri.

\section{ARACEAE}

Dracunculus vulgaris Schott, 34, 16.04.2014, BULU 35448, D. Akd., Kri.

\section{ASPARAGACEAE}

Asparagus acutifolius L., 30, 16.04.2014, BULU 35421, Akd., Fan.

Hyacinthella lineata (Steud. ex Schult. \& Schult f.) chouard, 11, 17.04.2015, BULU 38173, Kri., END., LC.

Muscari armeniacum Leichtlin ex Baker, 35, 23.06.2017, BULU 41582, Kri.

Muscari comosum (L.) Mill., 34, 30.05.2013, BULU 41341, Akd., Kri.

Muscari neglectum Guss. ex Ten., 9, 30.03.2013, BULU 40366, Kri.

Muscari tenuiflorum Tausch, 37, 24.05.2014, BULU 36448, Kri.

Ornithogalum armeniacum Baker, 12, 16.04.2014, BULU 35485, D. Akd., Kri.

Ornithogalum comosum L., 44, 22.05.2015, BULU 38275, Kri.

Ornithogalum nutans L., 6, 11.05.2016, BULU 39687, D. Akd., Kri.

Ornithogalum orthophyllum Ten., 13, 22.04.2014, BULU 35799, Kri.

Ornithogalum montanum Cirillo (Sin:O. platyphyllum Boiss.), 26, 22.04.2014, BULU 35743, D. Akd., Kri.

Ornithogalum sigmoideum Freyn \& Sint., 24, 30.03.2013, BULU 40342, Avr.-Sib., Kri.

Ornithogalum wiedemannii Boiss. var. wiedemannii, 26, 01.03.2016, BULU 39500, Kri.

Prospero autumnale (L.) Speta (Sin: Scilla autumnalis L.), 29, 24.09.2014, BULU 38001, Akd., Kri.

Ruscus aculeatus L., 30, 16.04.2014, BULU 35422, Kri.

15.CYPERACEAE

Carex caryophyllea Latourr. var. caryophyllea, 13, 22.04.2014, BULU 35790, Avr.-Sib., Kri.

Carex divisa Huds., 26, 22.04.2014, BULU 35750, Kri.

Carex flacca Schreb. subsp. erythrostachys (Hoppe) Holub (Sin: C. flacca Schreb. subsp. serrulata (Biv.) Greuter), 9, 17.04.2014, BULU 35537, Akd., Kri.

Carex melanostachya M. Bieb. ex Willd., 9, 16.05.2017, BULU 41404, Kri.

Carex pendula Huds., 26, 13.07.2016, BULU 40211, Avr.Sib., Kri.

Cyperus longus L. subsp. longus, 26, 13.07. 2016, BULU 40228, Kri.

\section{DIOSCOREACEAE}

Dioscorea communis (L.) Caddick \& Wilkin (Sin: Tamus communis L.), 6, 11.05.2016, BULU 39683, Kri.

\section{JUNCACEAE}

Juncus conglomeratus L., 10, 14.08.2015, BULU 39082, Avr.-Sib., Hem.

Juncus fontanesii J. Gay ex Laharpe subsp. fontanesii, 26, 13.07. 2016, BULU 40237, Kri.

Juncus inflexus L. subsp. inflexus, 26, 13.07. 2016, BULU 40239, Hem.

Juncus subulatus Forssk., 35, 30.07.2017, BULU 42039, Akd., Hem.

Luzula forsteri (Sm.) DC. subsp. caspica Novikov, 30, 16.04.2014, BULU 35422, Avr.-Sib., Kri.

\section{IRIDACEAE}

Crocus chrysanthus (Herb.) Herb., 26, 01.03.2016, BULU

39501, Kri.

Crocus flavus Weston subsp. dissectus T. Baytop \& B Mathew, 24, 12.03.2015, BULU 38071, Kri., END., VU.

Crocus pallasi Goldb. subsp. pallasi, 26, 06.11.2015, BULU 39490, Kri.

Crocus pulchellus Herb., 26, 06.11.2015, BULU 39489, Kri.

Iris kerneriana Asch. \& Sint. ex Baker, 8, 25.05.2016, BULU 39731, Avr.-Sib., Kri., END., LC.

Iris purpureobracteata B. Mathew \& T. Baytop, 8, 25.05.2016, BULU 39733, Kri., END., NT.

Iris suaveolens Boiss. \& Reut., 44, 17.04.2015, BULU 38115, Avr.-Sib., Kri.

Romulea linaresii Parl. subsp. graeca Beg., 26, 01.03.2016, BULU 39494, D. Akd., Kri.

\section{LILIACEAE}

Fritillaria pinardii Boiss., 45, 17.04.2015, BULU 38125 , Ir.-Tur., Kri.

Gagea chrysantha (Jan) Schult. \& Schult. f., 24, 30.03.2013, BULU 40336, Akd., Kri.

Tulipa orphanidea Boiss. ex Heldr., 43, 10.05.2011, BULU 40400, D. Akd., Kri.

\section{ORCHIDACEAE}

Cephalanthera epipactoides Fisch. \& C. A. Mey., 22, 22.05.2015, BULU 38332, D. Akd., Kri.

Dactylorhiza romana (Seb.) Soo subsp. romana, 18, 19.04.2016, BULU 39609, Akd., Kri.

Limodorum abortivum (L.) Sw. var. rubrum H. Sund. ex Kreutz, 13, 22.04.2014, BULU 35802, Kri.

Ophrys mammosa Desf. subsp. mammosa, 12, 22.04.2014, BULU 35786, Akd., Kri.

Ophrys oestrifera M. Bieb. subsp. oestrifera, 44, 24.05.2014, BULU 36418A, Kri.

Orchis palustris Jacq. subsp. palustris, 33, 16.04.2014, BULU 35382, Kri.

Orchis provincialis Balb. ex Lam. \& DC., 29, 16.04.2014, BULU 35444, Akd., Kri.

Orchis purpurea Huds. subsp. purpurea, 26, 25.06.2015, BULU 38379, Avr.-Sib., Kri.

Orchis simia Lam., 5, 09.05.2017, BULU 41211, Akd., Kri. 21.POACEAE

Aegilops geniculata Roth, 15, 11.06.2014, BULU 37230, Akd., Ter.

Aegilops triuncialis L. subsp. triuncialis, 24, 25.05.2014, BULU 36658, Ter.

Aira caryophyllea L., 5, 17.04.2014, BULU 35503, Avr.Sib., Ter.

Aira elegantissima Schur subsp. elegantissima, 43, 
11.05.2016, BULU 39660, Akd., Hem.

Agrostis canina L., 36, 10.07.2014, BULU 37629, Avr.Sib., Kri.

Agrostis capillaris L. var. capillaris, 30, 11.06.2014, BULU 37145, Hem.

Alopecurus myosuroides Huds. var. myosuroides, 9, 17.04.2014, BULU 35535B, Avr.-Sib., Hem.

Avena sativa L., 39, 10.07.2014, BULU 37622, Ter.

Brachypodium distachyon (L.) P. Beauv. (Sin: Trachynia distachya (L.) Link), 11, 25.05.2016, BULU 39702, Akd., Hem.

Brachypodium sylvaticum (Huds.) P. Beauv., 26, 25.06.2015, BULU 38437B, Avr.-Sib., Hem.

Briza maxima L., 27, 25.05.2014, BULU 36676, Ter.

Bromus hordeaceus L. subsp. hordeaceus, 37, 24.05.2014, BULU 36466, Ter.

Bromus japonicus Thunb. subsp. japonicus, 15, 11.06.2014, BULU 37229, Ter.

Bromus lanceolatus Roth, 24, 25.05.2014, BULU 36670B, Ter.

Bromus scoparius L., 26, 14.05.2015, BULU 38269, Ter. Bromus sterilis L., 9, 25.05.2014, BULU 36517, BULU 37408, Ter.

Bromus tectorum L., 26, 22.04.2014, BULU 35746, Ter. Calamagrostis pseudophragmites (Haller fil.) Koeler, 26, 25.06.2015, BULU 38437A, Avr.-Sib., Kri.

Catapodium rigidum (L.) C. E. Hubb. subsp. rigidum var. rigidum, 15, 11.06.2014, BULU 37232, Hem.

Cynosurus echinatus L., 29, 11.06.2014, BULU 37150, Akd., Ter.

Dactylis glomerata L. subsp. glomerata, 31, 11.06.2014, BULU 37124, Avr.-Sib., Hem.

Echinochloa colona (L.) Link, 21, 30.07.2017, 42066B, Hem.

Echinochloa crus-galli (L.) P. Beauv., 21, 30.07.2017, 42066A, Hem.

Eragrostis minor Host, 37, 24.05.2014, BULU 36465, Ter. Festuca callieri (Hack.) Markgr. subsp. callieri, 11, 17.04.2015, BULU 38170, Hem.

Festuca valesiaca Schleich. ex Gaudin, 29, 11.06.2014, BULU 37158, Hem.

Helictotrichon pubescens (Huds.) Schult. \& Schult. f. subsp. pubescens, 44, 14.05.2014, BULU 36053, Avr.-Sib., Hem.

Holcus lanatus L., 26, 23.06.2016, BULU 40201, Avr.Sib., Hem.

Hordeum geniculatum All., 31, 11.06.2014, BULU 37123, Avr.-Sib., Hem.

Lolium perenne L., 21, 30.07.2017, BULU 42065, Avr.Sib., Hem.

Lolium rigidum Gaudich var. rigidum, 8, 25.05.2016, BULU 39745, Ter.

Melica ciliata L. subsp. ciliata, 31, 11.06.2014, BULU 37107, Hem.

Melica uniflora Retz., 26, 25.06.2015, BULU 38404, Avr.Sib., Ter.

Phleum exaratum Griseb. subsp. exaratum, 27, 23.06.2017, BULU 41648, Ter.

Phleum pratense L., 46, 25.05.2014, BULU 36636A, Avr.Sib., Ter.

Phleum subulatum (Savi) Asch. \& Graebn. subsp. ciliatum (Boiss.) Humphries, 35, 23.06.2017, BULU 41573B, D. Akd., Ter.

Phragmites australis (Cav.) Trin. ex Steud., 21, 30.07.2017, BULU 42063, Avr.-Sib., Kri.

Poa alpina L. subsp. fallax F. Herm., 34, 16.04.2014, BULU 35465, Hem.

Poa bulbosa L., 30, 16.04.2014, BULU 35420, Kri.

Poa nemoralis L., 26, 22.04.2014, BULU 35747, Hem.

Poa supina Schrad., 38, 17.04.2014, BULU 35640, Avr.Sib., Kri.

Polypogon monspeliensis (L.) Desf., 21, 30.07.2017, BULU 42064, Hem.

Puccinellia festuciformis (Host) Parl., 26, 30.11.2012, BULU 40323, Ter.

Rostraria cristata (L.) Tzvelev var. cristata (Sin: Koeleria cristata (L.) Pers.), 8, 25.05.2016, BULU 39741, Hem.

Sorghum halepense (L.) Pers. var. halepense, 44, 09.07.2014, BULU 37551, Hem.

Taeniatherum caput-medusae (L.) Nevski subsp. crinitum (Schreb.) Melderis, 11, 25.05.2016, BULU 39701, İr.-Tur., Ter.

Tragus rasemosus (L.) All., 40, 21.07.2017, BULU 41676, Ter.

Vulpia ciliata Dumort subsp. ciliata, 37, 24.05.2014, BULU 36467, Ter.

Vulpia persica (Boiss. \& Buhse) Krecz \& Bobrov, 11, 25.05.2014, BULU 36548, İr.-Tur., Ter.

Vulpia unilateralis (L.) Stace, 44, 14.05.2014, BULU 36074, Ter.

\section{XANTHORRHOEACEAE}

Asphodeline taurica (Pall.) Endl., 26, 22.04.2014, BULU 35742, D. Akd., Kri.

Asphodeline lutea (L.) Rchb., 6, 19.04.2016, BULU 39627, Akd., Kri.

Sinif: Magnoliopsida (Dikotiller)

\section{ANACARDIACEAE}

Pistacia palaestina Boiss. (Sin: P. terebinthus L. subsp. palaestina (Boiss.) Engler), 35, 23.06.2017, BULU 41615, D. Akd., Fan.

Rhus coriaria L., 8, 19.04.2016, BULU 39621, Fan.

\section{APIACEAE}

Anthriscus nemorosa (M. Bieb.) Spreng., 30, 11.06.2014, BULU 37133, Hem.

Artedia squamata L., 44, 24.05.2014, BULU 36442, Hem. Bifora testiculata (L.) Spreng., 44, 22.05.2015, BULU 38276, Ter.

Buplerum sulphureum Boiss. \& Balansa, 44, 09.07.2014, BULU 37544, İr.-Tur.,Ter., END., LC.

Chaerophyllum byzantinum Boiss., 26, 25.06.2015, BULU 38414, İr.-Tur., Hem.

Conium maculatum L., 11, 25.05.2014, BULU 36541, Hem.

Coriandrum sativum L., 44, 14.05.2014, BULU 36072 , Ter.

Daucus carota L., 10, 27.06.2014, BULU 37380, Hem.

Daucus guttatus Sibter. \& Sm., 35, 23.06.2017, BULU 41536, Ter.

Elaeosticta aurea (Boiss.)Menemen (Sin: Muretia aurea Boiss.), 27, 23.06.2017, BULU 41657, Kri., END., VU.

Eryngium campestre L. var. virens Link, 20, 16.09.2012, BULU 40404, Hem. 
Ferulago macrosciadia Boiss. \& Balansa, 6, 11.05.2016, BULU 39674, D. Akd., Hem., END., LC.

Ferulago silaifolia (Boiss.) Boiss., 35, 30.07.2017, BULU 41999, İr.-Tur., Hem., END., EN.

Foeniculum vulgare Mill., 13, 24.05.2014, BULU 39699, Hem.

Heracleum platytaenium Boiss., 5, 11.05.2016, BULU 41244, İr.-Tur., Hem.

Johrenia dichotoma DC. (Sin: J. tortuosa (Fisch. \& C.A. Mey.) D. F. chamb.), 5, 23.06.2017, BULU 41663, İr.-Tur., Hem.

Laser trilobum (L.) Borkh., 26, 14.05.2015, BULU 38205, Hem.

Oenanthe silaifolia M. Bieb., 32, 11.06.2014, BULU 37104, Hem.

Orlaya daucoides (L.), 37, 24.05.2014, BULU 36464, Akd., Ter.

Scandix pecten-veneris L., 30, 16.04.2014, BULU 35403, Ter.

Scandix stellata Banks. \& Sol., 5, 16.05.2017, BULU 41230 A, Ter.

Smyrnium connatum Boiss. \& Kotschy, 27, 23.06.2017, BULU 41609, Akd., Hem.

Torilis arvensis (Huds.) Link subsp. elongata (Hoffmans \& Link) Cannon, 46, 09.07.2014, BULU 37433, D. Akd., Ter.

Torilis arvensis (Huds.) Link subsp. neglecta (Spreng.) Thell., 35, 23.06.2017, BULU 41583A, Ter.

Torilis leptophylla (L.) Rchb. f., 31, 11.06.2014, BULU 39099 B, Ter.

Torilis ucranica Spreng., 36, 10.07.2014, BULU 37633, Ter.

Turgeniopsis foeniculacea (Fenzl) Boiss., 36, 23.06.2017, BULU 41500, Ter.

\section{APOCYNACEAE}

Cionura erecta (L.) Griseb., 26, 25.06.2015, BULU 38383, D. Akd., Hem.

Cynanchum acutum L. subsp. acutum, 20, 16.09.2012, BULU 40402, Hem.

Periploca graeca L. var. graeca, 26, 25.06.2015, BULU 38382, D. Akd., Fan.

Vinca major L. subsp. major, 45, 22.05.2015, BULU 38305, Akd., Hem.

Vincetoxicum fuscatum Rchb. f. subsp. fuscatum, 26, 25.06.2015, BULU 38418, Hem.

Vincetoxicum tmoleum Boiss., 26, 25.06.2015, BULU 38431, İr.-Tur., Hem.

26.ARALIACEAE

Hedera helix L., 26, 14.05.2015, BULU 38207, Fan. 27.ARISTOLOCHIACEAE

Aristolochia bodamae Dingler, 28, 11.06.2014, BULU 37149, Hem.

Aristolochia pallida Willd., 30, 16.04.2014, BULU 35413, Hem.

28.ASTERACEAE

Achillea coarctata Poir., 3, 10.07.2014, BULU 37627, Hem.

Achillea nobilis L. subsp. neilreichii (A. Kern.) Velen., 27, 23.06.2017, BULU 41618A, Avr.-Sib., Hem.

Achillea pannonica Scheele (Sin: A. millefolium L. subsp. pannonica (Scheele) Oborny), 26, 16.06.2014, BULU
3624, Avr.-Sib., Hem.

Anthemis aciphylla Boiss. var. discoidea Boiss., 36, 10.07.2014, BULU 37628, D. Akd., Hem., END., LC.

Anthemis cotula L, 20, 23.06.2017, BULU 41546, Hem.

Anthemis cretica L. subsp. anatolica (Boiss.) Grierson, 45, 22.05.2015, BULU 38296, Hem.

Anthemis macrotis (Rech. f.) Oberpr. \& Vogt (Sin: Matricaria macrotis Rech.), 1, 22.05.2015, BULU 38350 , D. Akd., Ter.

Anthemis pseudocotula Boiss., 15, 11.06.2014, BULU 37219, Hem

Anthemis xylopoda O. Schwarz, 27, 23.06.2017, BULU 41221A, D. Akd., Hem., END., CR.

Arctium minus (Hill) Bernh., 5, 21.07.2017, BULU 41854, Avr.-Sib., Hem.

Asteriscus spinosus (L.) Sch. Bip., 11, 30.07.2017, BULU 41071, Akd., Ter.

Bellis perennis L., 24, 30.03.2013, BULU 40333, Avr.Sib., Hem.

Carduus acicularis Bertol., 31, 11.06.2014, BULU 37120, Akd., Ter.

Carduus nutans L. subsp. nutans, 11, 25.05.2016, BULU 39717, Hem

Carlina lanata L., 11, 30.07.2017, BULU 42073, Akd., Hem.

Carthamus dentatus (Forssk.)Vahl, 19, 30.07.2017, BULU 41964, Ter.

Centaurea diffusa Lam., 40, 21.07.2017, BULU 41693, Akd., Hem., END., NE.

Centaurea ertugruliana Uysal, 5, 11.05.2016, BULU 39695, Hem., END., CR.

Centaurea iberica Trev. ex Spreng., 11, 30.07.2017, BULU 42072, Hem.

Centaurea olympica (DC.) K.Koch, 35, 23.06.2017, BULU 41550, Hem., END., LC.

Centaurea solstitialis L. subsp. solstitialis, 40, 21.07.2017, BULU 41692, Hem.

Centaurea thracica (Janka) Hayek, 9, 25.05.2016, BULU 39754, Hem

Centaurea urvillei DC. subsp. urvillei, 44, 14.05.2014, BULU 36046, D. Akd., Hem.

Centaurea wiedemanniana Fisch. \& C.A.Mey., 44, 09.07.2014, BULU 37552, Hem., END., VU.

Cichorium intybus L., 8, 25.05.2016, BULU 39768, Hem. Cirsium hypoleucum DC., 5, 21.07.2017, BULU 41855 Avr.-Sib., Hem.

Cnicus benedictus L., 12, 22.04.2014, BULU 35785, Hem. Conyza canadensis (L.) Cronquist, 26, 30.11.2012, BULU 40321, Hem.

Cota altissima (L.) J. Gay (Sin: Anthemis altissima L.), 46, 25.05.2014, BULU 36620 B, Ter.

Cota tinctoria (L.) J. Gay ex Guss. var. pallida (DC.) Özbek \& Vural (Sin: Anthemis tinctoria L. var. pallida DC.), 13, 27.06.2014, BULU 37340, Hem.

Cota tinctoria (L.) J.Gay ex Guss. var. tinctoria (Sin: Anthemis tinctoria L. var. tinctoria), 28, 16.04.2014, BULU 35431, Hem

Cota triumfetti (L.) J. Gay ex Guss. (Sin: Anthemis triumfettii (L.) All.), 37, 24.05.2014, BULU 36474, Hem. Crepis commutata (Spreng.) Greuter (Sin: C. foetida L. subsp. commuta (Spreng.) Babc.), 37, 24.05.2014, BULU 
36462, Hem.

Crepis macropus Boiss. \& Heldr., 24, 25.05.2014, BULU 36669B, İr.-Tur., Hem., END., LC.

Crepis sancta (L.) Bornm., 35, 23.06.2017, BULU 41525, Ter.

Crepis setosa Haller f., 11, 25.05.2016, BULU 39700, Avr.-Sib., Ter.

Crepis zacintha (L.) Babc., 9, 17.04.2014, BULU 35520, Akd., Ter.

Crupina crupinastrum (Moris) Vis., 44, 14.05.2014, BULU 36075, Ter.

Cyanus depressus (M.Bieb.) Sojak (Sin: Centaurea depressa M. Bieb. var. amasiana Bormn.), 38, 17.04.2014, BULU 35646, Ter.

Cyanus segetum Hill (Sin: Centaurea cyanus), 36, 23.06.2017, BULU 41508, Ter.

Cyanus thirkei (Sch. Bip.) Holub (Sin: Centaurea thirkei Sch. Bip. ), 9, 17.04.2014, BULU 35534, D. Akd., Hem.

Cyanus triumfettii Dostal ex A. Löve \& D. Löve subsp. triumfettii (Sin: Centaurea triumfettii All.) , 45, 22.05.2015, BULU 38303, Hem.

Doronicum orientale Hoffm., 30, 16.04.2014, BULU 35397, Hem.

Echinops spinosissimus Turra subsp. spinosissimus (Sin: E. viscosus DC. subsp. viscosus), 35, 23.06.2017, BULU 41575, D. Akd., Hem.

Filago germanica (L.) L. (Sin: F. vulgaris Lam.), 44, 24.05.2014, BULU 36417, Hem.

Helichrysum luteo-album (L.) Rchb. (Sin: Gnaphalium luteo-album L.), 36, 23.06.2017, BULU 41490, Hem.

Hieracium pannosum Boiss., 19, 30.07.2017, BULU 41972, D. Akd., Hem.

Inula graveolens (L.) Desf., 35, 25.09.2017, BULU 42080, D.Akd., Ter.

Inula heterolepis Boiss., 27, 12.03.2015, BULU 38088, D. Akd., Hem.

Jurinea consanguinea DC., 1, 22.05.2015, BULU 38357, Hem.

Leontodon asperrimus (Willd.) Endl., 27, 10.07.2014, BULU 37619, İr.-Tur., Hem.

Leontodon crispus Vill. subsp. asper (Waldst. \& Kit.) Röhl. var. asper, 45, 22.05.2015, BULU 38310, Hem.

Leontodon hispidus L. var. hispidus, 29, 16.04.2014, BULU 35439, Hem.

Matricaria chamomilla L. var. chamomilla, 27, 23.06.2017, BULU 41621 B, Ter.

Matricaria chamomilla L. var. recutita (L.) Fiori, 37, 24.05.2014, BULU 36474, Ter.

Onopordum illyricum L., 40, 21.07.2017, BULU 41673, D. Akd., Hem.

Onopordum tauricum Willd., 27, 23.06.2017, BULU 41654, Avr.-Sib., Hem.

Picris pauciflora Willd., 31, 11.06.2014, BULU 37117B, Akd., Ter.

Pilosella hoppeana (Schult.) F.W. Schultz \& Sch. Bip subsp. testimonialis (Naegli ex Peter) P. D. Sell \& C. West (Sin: P. hoppeana subsp. lydia (Bornm. \& Zahn) P. D. Sell \& C. West), 36, 10.07.2014, BULU 37634, Hem.

Pilosella piloselloides (Vill.) Sojak subsp. magyarica (Peter) S. Braut. \& Greuter (Sin: P. piloselloides subsp. megalomastix (Nageli \& Peter) P. D. Sell \& C. West), 45,
22.05.2015, BULU 38311, Hem.

Pilosella piloselloides (Vill.) Sojak subsp. piloselloides, 26, 16.06.2014, BULU 36249, Hem.

Pulicaria dysentarica (L.) Bernh. subsp. dysentarica, 35, 30.07.2017, BULU 42019, Hem.

Rhagadiolus stellatus (L.) Gaertn. (Sin: R. stellatus var. edulis (Gaertn.) DC.), 1, ,22.05.2015, BULU 38367, Akd., Ter.

Scorzonera cana (C. A. Mey.) Griseb. var. jacquiniana (W. Koch) D. F. Chamb., 45, 22.05.2015, BULU 38319, Hem. Scorzonera eriophora DC., 17, 19.04.2016, BULU 39591, Hem., END., LC.

Scorzonera mollis M.Bieb. subsp. mollis, 45, 22.05.2015, BULU 38319, Hem.

Senecio jacobaea L., 27, 12.03.2015, BULU 38086, Ter. Senecio vernalis Waldst. \& Kit., 12, 16.04.2014, BULU 35491, Ter.

Senecio vulgaris L., 36, 28.03.2017, BULU 41099, Ter. Sonchus asper (L.) Hill. subsp. glaucescens (Jord.) Ball, 26, 25.06.2015, BULU 38427, Hem.

Tanacetum parthenium (L.) Sch. Bip., 26, 23.06.2016, BULU 40212, Hem.

Taraxacum hellenicum Dahlst., 18, 19.04.2016, BULU 39613, Hem.

Taraxacum pseudobrachyglossum Soest, 33, 16.04.2014, BULU 35387, Avr.-Sib., Hem., END., NT.

Taraxacum scaturiginosum G. E. Haglund, 44, 17.04.2015, BULU 38109B, Hem.

Taraxacum serotinum (Waldst. \& Kit.) Poir., 26 , 22.04.2014, BULU 35716, Hem.

Tragopogon bupthalmoides (DC.) Boiss. var. bupthalmoides, 44, 14.05.2014, BULU 36068, İr-Tur., Hem.

Tragopogon dubius Scop., 24, 25.05.2014, BULU 36617 B Hem.

Tripleurospermum tenuifolium (Kit.) Freyn, 26, 22.04.2014, BULU 35717, Avr.-Sib., Hem.

Tussilago farfara L., 26, 01.03.2016, BULU 39496, Hem. Urospermum picroides (L.) Scop. ex F. W. Schmidt, 31, 11.06.2014, BULU 37117A, Akd., Ter.

Xanthium orientale L. subsp. italicum (Moretti) Greuter (Sin: X. strumarium L. subsp. cavanillesii (Schouw) D.Löve \& P. Dansereau, 21, 30.07.2017, BULU 42047, Ter.

Xanthium spinosum L., 21, 30.07.2017, BULU 42049 Ter.

Xeranthemum annuum L., 39, 10.07.2014, BULU 37618, Ter.

29.BETULACEAE

Alnus glutinosa (L.) Gaertn. subsp. glutinosa, 26, 07.04.2016, BULU 39551, Avr.-Sib., Fan.

Carpinus betulus L., 4, 26.03.2014, BULU 35249, Fan. 30.BORAGINACEAE

Aegonychon purpurocaeruleum (L.) Holub (Sin: Lithospermum purpurocaeruleum L., 17, 19.04.2016, BULU 39595, Avr.-Sib., Hem.

Alkanna orientalis (L.) Boiss. var. leucantha (Born.) HubMor., 12, 16.04.2014, BULU 35487, Ir.-Tur., Hem., END., NT.

Anchusa hybrida Ten. (Sin: A. undulata subsp. hybrida (Ten.) Beg.), 14, 19.04.2017, BULU 41200, Akd., Hem. 
Anchusa leptophylla Roem. \& Schult. subsp. leptophylla, 39, 08.06.2017, BULU 41415, Hem.

Anchusa officinalis L., 26, 25.06.2015, BULU 38407, Avr.Sib., Hem.

Buglossoides arvensis (L.) I. M. Johnst. subsp. sibthorpiana (Griseb.) R. Fern., 28, 16.04.2014, BULU 35430, Ter.

Buglossoides incrassata (Guss.) I. M. Johst. subsp. incrassata, 44, 24.05.2014, BULU 36420, Akd., Ter.

Cerinthe minor L. subsp. auriculata (Ten.) Domac, 1, 22.05.2015, BULU 38372, Hem.

Cynoglottis barrelieri (All.) Vural \& Kit Tan (Sin: Anchusa barrellieri (All.) Vitman var. orientalis Guşuleac), 15, 19.04.2016, BULU 39570, D. Akd., Hem.

Echium italicum L., 24, 25.05.2014, BULU 36657, Akd., Hem.

Heliotropium hirsutissimum Grauer, 10, 14.08.2015, BULU 39081, D. Akd., Ter.

Heliotropium suaveolens M. Bieb., 35, 23.06.2017, BULU 41614, D. Akd., Ter.

Myosotis arvensis (L.) Hill, 37, 24.05.2014, BULU 36454, Avr.-Sib.., Hem.

Myosotis incrassata Guss., 44, 17.04.2015, BULU 381010, D. Akd., Ter.

Myosotis ramosissima Rochel., 25, 22.04.2014, BULU 35765, Ter.

Neatostema apulum (L.) I. M. Johnst., 11, 30.07.2017, BULU 42077, Akd., Ter.

Onosma taurica Willd. var. taurica, 8, 19.04.2016, BULU 39622, Hem.

Onosma thracica Velen., 44, 22.05.2015, BULU 38287, Avr.-Sib., Hem.

Paracaryum calycinum Boiss. \& Balansa, 45, 22.05.2015, BULU 38301, İr.-Tur., Hem., END., LC.

Symphytum orientale L., 26, 14.05.2015, BULU 38231, Avr.-Sib., Hem.

Trachystemon orientalis (L.) G. Don, 26, 14.05.2015, BULU 38204, İr.-Tur., Kri.

\section{BRASSICACEAE}

Aethionema arabicum (L.) Adrz. ex DC., 8, 19.04.2016, BULU 39619, Ter.

Aethionema cordatum (Desf.) Boiss., 45, 22.05.2015, BULU 38315, Hem.

Alliaria petiolata (M. Bieb.) Cavara \& Grande, 26, 22.04.2014, BULU 35649, Ter.

Alyssum alyssoides (L.) L., 38, 17.04.2014, BULU 35632, Ter.

Alyssum cassium Boiss., 46, 25.05.2014, BULU 36601, D. Akd., Hem.

Alyssum corsicum Duby, 11, 25.05.2016, BULU 39698, Hem.

Alyssum dudleyi Adıgüzel \& R. D. Reeves, 45, 22.05.2015, BULU 38320, Hem., END., VU.

Alyssum hirsutum M.Bieb. subsp. hirsutum, 33, 16.04.2014, BULU 35385, Ter.

Alyssum minutum Schlecht. ex DC., 30, 16.04.2014, BULU 35407, Ter.

Alyssum murale Waldst. \& Kit. subsp. murale var. murale, 31, 11.06.2014, BULU 37108, Hem.

Alyssum sibiricum Willd., 26, 14.05.2015, BULU 38200, Hem.
Alyssum simplex Rudolph (Sin: A. minus Rothm.), 9, 17.04.2014, BULU 35538, Ter.

Alyssum strigosum Banks \& Sol. subsp. strigosum, 12, 16.04.2014, BULU 35490, Ter.

Alyssum strictum Willd., 25, 25.05.2014, BULU 36666, İr.-Tur., Ter.

Alyssum umbellatum Desv., 29, 16.04.2014, BULU 35441, D. Akd., Hem.

Arabidopsis thaliana (L.) Heynh., 37, 24.05.2014, BULU 36451, Ter.

Arabis alpina L. subsp. alpina (Sin: A. caucasica Willd. subsp. caucasica), 26, 07.04.2016, BULU 39531, Hem.

Aubrieta olympica Boiss., 27, 12.03.2015, BULU 38093, Hem., END., VU.

Aurinia saxatilis (L.) Desv. subsp. orientalis (Ard.) T. R Dudley, 26, 22.04.2014, BULU 35677, Hem.

Brassica nigra (L.) W. D. J. Koch, 36, 23.06.2017, BULU 41499, Ter.

Calepina irregularis (Asso) Thell., 23, 30.03.2013, BULU 40351, Ter.

Capsella bursa-pastoris (L.) Medik., 23, 30.03.2013, BULU 40357, Kozm., Ter.

Cardamine graeca L., 26, 22.04.2014, BULU 35661, Ter. Cardamine hirsuta L., 30, 16.04.2014, BULU 35418, Ter. Clypeola jonthlaspi L., 33, 16.04.2014, BULU 35384, Ter. Descurania sophia (L.) Webb ex Prantl subsp. sophia, 11, 25.05.2016, BULU 39718, Ter.

Draba muralis L., 12, 16.04.2014, BULU 35489, Ter.

Draba verna L. (Sin: Erophila verna L.), 41, 28.04.2013, BULU 35398, Ter.

Erysimum crassipes Fisch. \& C. A. Mey., 44, 22.05.2015, BULU 38293, Hem.

Erysimum graecum Boiss. \& Heldr., 37, 24.05.2014, BULU 36476, Hem.

Hesperis bicuspidata (Willd.) Poir., 6, 19.04.2016, BULU 39629, İr.-Tur., Hem., END., NE.

Isatis tinctoria L. subsp. tinctoria, 26, 25.06.2015, BULU 38428, Hem

Isatis tinctoria subsp. tomentella (Boiss.) P. H. Davis, 1, 22.05.2015, BULU 38342, Hem.

Lepidium draba L. (Sin: Cardaria draba (L.) Desv.), 39, 08.06.2017, BULU 41414, Hem.

Microthlaspi perfoliatum (L.) F. K. Mey. (Sin: Thlaspi perfoliatum L.), 29, 16.04.2014, BULU 35435, Ter.

Raphanus raphanistrum L. subsp. raphanistrum, 36, 23.06.2017, BULU 41498, Ter.

Rapistrum rugosum (L.) All., 11, 25.05.2016, BULU 39722, Ter.

Sisymbrium altissimum L., 39, 08.06.2017, BULU 41412, Ter.

Sisymbrium officinale (L.) Scop., 37, 24.05.2014, BULU 36171, Ter.

Rorippa sylvestris (L.) Besser subsp. sylvestris, 26, 13.07.2016, BULU 40240, Hem.

Teesdalia coronopifolia (Berg.) Thellung, 28, 16.04.2014, BULU 35429, D. Akd., Ter.

Thlaspi arvense L., 33, 16.04.2014, BULU 35380, Ter. 32.CAMPANULACEAE

Asyneuma linifolium (Boiss. \& Heldr.) Bornm. subsp. linifolium, 13, 27.06.2014, BULU 37343, D. Akd., Hem., END., LC. 
Asyneuma virgatum (Labill.) Bornm. subsp. cichoriiforme (Boiss.) Damboldt, 31, 11.06.2014, BULU 37099A, D. Akd., Hem.

Campanula betonicifolia Sm. \& Sm. subsp. betonicifolia, 6, 11.05.2016, BULU 39677, Hem., END., NT.

Campanula cymbalaria Sibter. \& Sm., 26, 14.05.2015, BULU 38230, Hem.

Campanula lyrata Lam. subsp. lyrata, 8, 25.05.2016, BULU 39760, Hem.

Campanula persicifolia L. subsp. persicifolia, 35, 23.06.2017, BULU 41528, Hem.

Legousia pentagonia (L.) Thell., 25, 25.05.2014, BULU 36644, D. Akd., Ter.

Legousia speculum-veneris (L.) Durande ex Vill., 5, 16.05.2017, BULU 41227, Ter.

\section{CANNABACEAE}

Celtis australis L. subsp. australis, 19, 30.07.2017, BULU 41981, Akd., Fan.

\section{CAPRIFOLIACEAE}

Cephalaria transsylvanica (L.) Schrad., 7, 21.07.2017, BULU 41827, Hem.

Dipsacus laciniatus L., 19, 30.07.2017, BULU 41982, Hem.

Knautia degenii Borbas ex Formanek, 9, 25.05.2014, BULU 36501, Akd., Hem.

Knautia integrifolia (L.) Bert. var. bidens (Sm.) Borbas, 1, 22.05.2015, BULU 38373, D. Akd., Ter.

Knautia orientalis L., 10, 27.06.2014, BULU 37372, D.

Akd., Ter.

Scabiosa argentea L., 36, 02.09.2014, BULU 37985B, Hem.

Scabiosa atropurpurea L. (Sin: S. atropurpurea L. subsp. maritima (L.) Are.), 21, 30.07.2017, BULU 42048, Hem.

Scabiosa columbaria L. subsp. columbaria var. columbaria, 9, 25.05.2014, BULU 36508, Hem.

Scabiosa reuteriana Boiss., 15, 11.06.2014, BULU 37240, D. Akd., Ter., END., LC.

Scabiosa triniifolia Friv., 5, 25.07.2016, BULU 40039B, Hem.

Pterocephalus plumosus (L.) Coulter, 31, 11.06.2014, BULU 37106, Ter.

Valeriana dioscoridis Sm., 17, 19.04.2016, BULU 39593, D. Akd., Kri.

Valerianella carinata Loisel., 27, 07.04.2016, BULU 39532, Ter.

Valerianella glomerata Boiss. \& Balansa, 35, 23.06.2017, BULU 41542, Ir.-Tur., Ter., END., LC.

Valerianella lasiocarpa Steven ex Betcke, 30, 16.04.2014, BULU 35412, İr.-Tur., Ter.

Valerianella pumila (L.) DC., 11, 17.04.2015, BULU 39603, Ter.

Lonicera etrusca Santi var. etrusca, 46, 25.04.2014, BULU 36622, Akd., Fan.

\section{CARYOPHYLLACEAE}

Agrostemma githago L., 36, 23.06.2017, BULU 41504, Ter.

Arenaria filicaulis Fenzl subsp. filicaulis, 26, 25.06.2015, BULU 38395, Hem.

Arenaria serpyllifolia L. subsp. leptoclados (Rchb.) Nyman (Sin: A. leptoclados (Reichb.) Guss., 37, 24.05.2014, BULU 36453, Ter.
Cerastium banaticum (Rochel) Steud. subsp. banaticum, 30, 16.04.2014, BULU 35417, Kam.

Cerastium brachypetalum Pers. subsp. roeseri (Boiss. \& Heldr.) Nyman, 17, 19.04.2016, BULU 39602, Ter.

Cerastium comatum Desv. (Sin: C. illyricum Ard. subsp. comatum (Desv.) P. D. Sell \& Whitehead, 34, 16.04.2014, BULU 35452, Akd., Ter.

Cerastium glomeratum Thuill., 23, 30.03.2013, BULU 40352, Ter.

Cerastium gracile Dufour, 45, 17.04.2015, BULU 38118, Ter.

Dianthus anatolicus Boiss., 17, 30.07.2017, BULU 41973, Hem.

Dianthus carmelitarum Reut. ex Boiss. (Sin. D. artwinensis Grossh.), 36, 02.09.2014, BULU 37980, Avr.Sib., Hem., END., LC.

Dianthus calocephalus Boiss., 15, 11.06.2014, BULU 37236, Hem

Dianthus cibrarius Clementi, 35, 23.06.2017, BULU 41557, Hem., END., NT.

Dianthus leptopetalus Willd., 45, 23.06.2017, BULU 41611, Hem.

Dianthus giganteus d'Urv., 30, 11.06.2014, BULU 37127, Avr.-Sib., Hem.

Dianthus goekayi Kaynak, Yılmaz \& Daşkın, 10, 14.08.2015, BULU 39083, Hem., END., CR.

Herniaria glabra L., 26, 16.06.2014, BULU 36253, Hem. Herniaria micrantha A.K. Jacks. \& Turrill, 35, 23.06.2017, BULU 41523, D. Akd., Hem.

Holosteum umbellatum L. var. umbellatum, 34, 16.05.2017, BULU 41331, Ter.

Minuartia hirsuta (M. Bieb.) Hand.-Mazz. subsp. falcata (Gris.) Mattf., 11, 17.04.2015, BULU 38163, Hem.

Minuartia hybrida (Vill.) Schischk. subsp. hybrida, 26 14.05.2015, BULU 38198, Ter.

Minuartia leucocephaloides (Bornm.) Bornm., 26, 22.04.2014, BULU 35703, Hem., END., LC.

Moenchia mantica (L.) Bartl., 46, 16.05.2017, BULU 41297, Ter.

Paronychia chionaea Boiss. subsp. chionaea var. chionaea, 45, 22.05.2015, BULU 38321, Hem.

Petrorhagia cretica (L.) P. W. Ball \& Heywood, 44, 09.07.2014, BULU 37547, Ter.

Petrorhagia dubia (Raf.) G. Lopez \& Romo (Sin: P. velutina (Guss.) P. W. Ball \& Heywood), 34, 11.06.2014, BULU 37178, Ter.

Petrorhagia prolifera (L.) P. W. Ball \& Heywood, 39, 10.07.2014, BULU 37611, Ter.

Saponaria glutinosa M. Bieb., 5, 11.05.2016, BULU 39690, Hem

Saponaria officinalis L., 26, 23.06.2016, BULU 40223, Hem.

Scleranthus annuus L. subsp. annuus, 47, 11.06.2014, BULU 41336, Ter.

Scleranthus annuus L. subsp. verticillatus (Tausch) Arcang., 37, 24.05.2014, BULU 36461, Ter.

Silene argentea Ledeb. (Sin: S. cappadocica Boiss. \& Heldr.), 13, 27.06.2014, BULU 37347, Ir.-Tur., Hem.

Silene compacta Fisch. ex Hornem., 35, 23.06.2017, BULU 41608, Hem.

Silene conoidea L., 37, 24.05.2014, BULU 36475, Ter. 
Silene dichotoma Ehrh. subsp. dichotoma, 39, 08.06.2017, BULU 41400, Hem.

Silene italica (L.) Pers. subsp. italica, 34, 16.05.2017, BULU 41344, Akd., Hem.

Silene latifolia Poir. subsp. eriocalycinae (Boiss.) Greuter \& Burdet (Sin: S. alba subsp. eriocalycina (Boiss.) Walters), 5, 16.05.2017, BULU 41235, Hem.

Silene supina M.Bieb. subsp. pruinosa (Boiss.) Chowdhuri, 25, 22.04.2014, BULU 35764, Hem.

Spergula pentandra L., 27, 07.04.2016, BULU 39522, Ter. Stellaria media (L.) Vill., 24, 30.03.2013, BULU 40332, Ter.

Vaccaria hispanica (Mill.) Rauschert (Sin: V. pyramidata Medik.), 11, 25.05.2016, BULU 39721, Ter.

Velezia rigida L., 36, 10.07.2014, BULU 37637, Ter.

\section{CHENOPODIACEAE}

Chepodium album L. subsp. album var. microphyllum Boenn. (Sin: C. album L. subsp. microphyllum (Boenn.) Sterner), 40, 21.07.2017, BULU 41688, Ter.

Chenopodium murale L., 27, 23.06.2017, BULU 41644 A, Ter.

Chenopodium vulvaria L., 27, 23.06.2017, BULU 41644B, Ter.

\section{CISTACEAE}

Cistus creticus L., 9, 25.05.2014, BULU 36501, Kam. Cistus salviifolius L., 13, 22.04.2014, BULU 35789, Kam. Helianthemum nummularium (L.) Mill. subsp. nummularium, 22, 19.04.2016, BULU 39607, Kam.

Helianthemum salicifolium (L.) Mill., 44, 14.05.2014, BULU 36131A, Ter.

Fumana aciphylla Boiss., 45, 22.05.2015, BULU 38294, Ir.-Tur., Kam.

Fumana thymifolia (L.) Spach, 44, 24.05.2014, BULU 36424, Kam.

\section{CLEOMEACEAE}

Cleome ornithopodioides L., 36, 02.09.2014, BULU 37994, D. Akd., Ter.

\section{CONVOLVULACEAE}

Convolvulus arvensis L., 40, 21.07.2017, BULU 41681, Kozm., Ter.

Convolvulus cantabrica L., 44, 22.05.2015, BULU 38271, Akd., Hem.

Convolvulus compactus Boiss., 8, 25.05.2016, BULU 39771, İr.-Tur., Hem.

Convolvulus holosericeus M.Bieb. subsp. holosericeus, 44, 24.05.2014, BULU 36441, İr.-Tur., Hem.

\section{CORNACEAE}

Cornus mas L., 4, 26.03.2014, BULU 35251, Avr.-Sib., Fan.

\section{CORYLACEAE}

Corylus avellana L. var. avellana, 26, 25.06.2015, BULU 38377, Avr.-Sib., Fan.

42.CRASSULACEAE

Umbilicus luteus (Huds.) Webb. \& Berthel. (Sin: U. erectus DC.), 12, 16.04.2014, BULU 35484, Hem.

Sedum acre L. subsp. acre, 31, 11.06.2014, BULU 37115, Hem.

Sedum album L., 13, 27.06.2014, BULU 37346, Hem.

Sedum hispanicum L., 27, 16.06.2014, BULU 36245, İr.Tur., Hem.

Phedimus obtusifolius (C.A. Mey.) 't Hart (Sin. Sedum obtusifolium C.A.Mey.), 35, 23.06.2017, BULU 41616, Hem.

Sedum pallidum M.Bieb. (Sin: S. pallidum M. Bieb. var. bithynicum (Boiss.) D. F. chamb.), 12, 16.04.2014, BULU 35495, Ir.-Tur., Hem.

43.ERICACEAE

Arbutus andrachne L., 3, 26.03.2014, BULU 35248, Fan. Arbutus unedo L., 9, 25.05.2014, BULU 36520, Fan.

Erica arborea L., 3, 26.03.2014, BULU 35244, Fan.

44.EUPHORBIACEAE

Euphorbia aleppica L., 21, 30.07.2017, BULU 42061, Ter. Euphorbia anacampceros Boiss. var. anacampceros, 38, 17.04.2014, BULU 35636, Hem., END., LC.

Euphorbia apios L., 33, 16.04.2014, BULU 35379, D. Akd., Hem.

Euphorbia falcata L. subsp. falcata var. galilaea (Boiss.) Boiss., 8, 19.04.2016, BULU 39615, Ter.

Euphorbia falcata L. subsp. macrostegia (Bornm.) O. Schwartz, 35, 30.07.2017, BULU 42006, D. Akd., Ter.

Euphorbia helioscopia L. subsp. helioscopia, 43, 10.05.2011, BULU 40401, Ter.

Euphorbia herniariifolia Willd. var. glaberrima Halacsy, 5, 11.05.2016, BULU 39694, Hem.

Euphorbia prostrata Aiton, 7, 21.07.2017, BULU 41833, Ter.

Euphorbia taurinensis All., 11, 17.04.2015, BULU 38168, Ter.

Mercurialis annua L., 23, 30.03.2013, BULU 40362B, Ter. Mercurialis perennis L., 26, 22.04.2014, BULU 35691, Avr.-Sib., Hem.

45.FABACEAE

Astragalus gaeobotrys Boiss. \& Balansa, 34, 16.04.2014, BULU 35458; 15, 19.04.2016, BULU 39569, Hem., END., EN.

Astragalus coodei D. F. Chamb. \& V. A. Mathews, 17, 19.04.2016, BULU 39583, İr.-Tur., Hem., END., LC.

Astragalus hamosus L., 15, 11.06.2014, BULU 37224, Ter.

Astragalus glycyphyllos L., 46, 25.05.2014, BULU 36615, Avr.-Sib., Hem.

Astragalus vulnerariae DC., 45, 22.05.2015, BULU 38300, Hem., END., LC.

Biserrula pelecinus L., 37, 24.05.2014, BULU 36450, Akd., Ter.

Bituminaria butiminosa (L.) C.H.Stirt (Sin: Psoralea butiminosa L.), 35, 10.06.2016, BULU 39963, Akd., Hem. Cercis siliquastrum L. subsp. siliquastrum, 18, 19.04.2016, BULU 39611, Akd., Fan.

Colutea cilicica Boiss. \& Balansa, 15, 11.06.2014, BULU 36228, Fan.

Coronilla scorpioides (L.) W. D. J. Koch, 12, 22.04.2014, BULU 35777 B, Ter.

Cytisus hirsutus L. (Sin: Chamaecytisus hirsutus (L.) Link), 30, 16.04.2014, BULU 35415, Kam.

Cytisus pygmaeus Willd. (Sin: Chamamaecytisus pygmaeus (Willd.) Rothm., 13, 22.04.2014, BULU 35798, Avr.-Sib., Kam.

Cytisopsis dorycniifolia Jaub. \& Spach, 15, 19.04.2016, BULU 39568, Kam.

Dorycnium graecum (L.) Ser., 30, 11.06.2014, BULU 37169A, İr.-Tur., Hem. 
Dorycnium pentaphyllum Scop. subsp. herbaceum (Vill.) Rouy, 8, 25.05.2016, BULU 39752, Hem.

Hymenocarpus circinnatus (L.) Savi, 1, 22.05.2015, BULU 38371, Ter.

Lathyrus digitatus (M. Bieb.) Fiori, 38, 17.04.2014, BULU 35644, D. Akd., Hem.

Lathyrus laxiflorus (Desf.) O. Kuntze subsp. laxiflorus, 34, 16.04.2014, BULU 35456, Hem.

Lathyrus sativus L., 34, 16.04.2014, BULU 35457, Akd., Hem.

Lathyrus undulatus Boiss., 30, 16.04.2014, BULU 35396, İr.-Tur., Hem., END., VU.

Lotus angustissimus L., 29, 24.09.2014, BULU 37999, Ter.

Lotus corniculatus L. var. corniculatus, 10, 27.06.2014, BULU 37375, Hem.

Medicago coronata (L.) Bart., 44, 09.07.2014, BULU 37545, Akd., Ter.

Medicago lupulina L., 12, 22.04.2014, BULU 35782, Ter. Medicago minima (L.) Bartal. var. minima, 12, 22.04.2014, BULU 35782, Ter.

Medicago orbicularis (L.) Bartal., 1, 22.05.2015, BULU 38354, Ter.

Medicago sativa L. subsp. sativa, 12, 16.04.2014, BULU 36716, Hem.

Melilotus officinalis (L.) Desr., 7, 21.07.2017, BULU 41839, Ter.

Onobrychis aequidentata (Sibth \& Sm.) d'Urv, 9, 25.05.2014, BULU 39762, Akd., Ter.

Onobrychis caput-galli (L.) Lam., 5, 25.05.2014, BULU 36712, Akd., Ter.

Ononis pusilla L., 1, 22.05.2015, BULU 38369, Akd., Hem.

Ornithopus compressus L., 37, 24.05.2014, BULU 36455, Akd., Ter.

Scorpiurus subvillosus L. var. subvillosus (Sin: S. muricatus L. var. subvillosus (L.) Fiori), 1, 22.05.2015, BULU 38370, Ter.

Securigera cretica (L.) Lassen, 12, 22.04.2014, BULU 35776, Akd., Ter.

Securigera varia (L.) Lassen (Sin: Coronilla varia L. var. varia), 30, 11.06.2014, BULU 37166A, D. Akd., Hem.

Trifolium alpestre L. var. alpestre, 6, 11.05.2016, BULU 39672, Avr.-Sib., Hem.

Trifolium angustifolium L., 29, 11.06.2014, BULU 37157, Hem.

Trifolium arvense L. var. arvense, 9, 25.05.2014, BULU 36520, Avr.-Sib., Ter.

Trifolium campestre Schreb. subsp. campestre var. campestre, 29, 11.06.2014, BULU 37153, Ter.

Trifolium diffusum Ehrh., 9, 25.05.2014, BULU 36507, Ter.

Trifolium echinatum M. Bieb., 24, 25.05.2014, BULU 36651; 34, 11.06.2014, BULU 37121, Ter.

Trifolium grandiflorum Schreb. (Sin: T. speciosum Willd.), 6, 11.05.2016, BULU 39673, D. Akd., Ter.

Trifolium hirtum All., 9, 25.05.2014, BULU 36514, Akd., Ter.

Trifolium lappaceum L., 28, 11.06.2014, BULU 37156, Akd., Ter.

Trifolium nigrescens Viv. subsp. nigrescens, 5,
11.05.2016, BULU 39678, Ter.

Trifolium nigrescens subsp. petrisavii (Clementi) Holmboe, 25, 25.05.2014, BULU 36665, Ter.

Trifolium pallidum Waldst. \& Kit., 36, 10.07.2014, BULU 37638, Ter.

Trifolium patens Schreb., 36, 22.05.2015, BULU 38325, Ter.

Trifolium purpureum Lois. var. purpureum, 11, 25.05.2016, BULU 39706, D. Akd., Ter.

Trifolium repens L. var. repens, 29, 11.06.2014, BULU 37148, Hem.

Trifolium resupinatum L. var. resupinatum, 28, 11.06.2014, BULU 37148, Ter.

Trifolium scabrum L., 31, 11.06.2014, BULU 37121, Akd., Ter.

Trifolium stellatum L. var. stellatum, 24, 25.05.2014, BULU 36672A, Ter.

Vicia cracca L. subsp. cracca, 1, 22.05.2015, BULU 38345, Avr.-Sib., Hem.

Vicia cretica Boiss. \& Heldr., 8, 19.04.2016, BULU 39618, Ter.

Vicia grandiflora Scop., 6, 17.04.2014, BULU 35504, Ter. Vicia hirsuta (L.) Gray, 43, 11.05.2016, BULU 39658, Ter.

Vicia hybrida L., 12, 22.04.2014, BULU 35779, Ter.

Vicia lathyroides L., 30, 16.04.2014, BULU 35392, Ter. Vicia meyeri Boiss., 34, 16.04.2014, BULU 35459, İr.Tur., Ter.

Vicia sativa L. subsp. sativa, 13, 22.04.2014, BULU 35794, Ter.

Vicia villosa Roth subsp. dasycarpa (Ten.) Cav., 46, 25.05.2014, BULU 36640A, Ter.

Vicia villosa Roth subsp. eriocarpa (Hausskn.) P. W. Ball, 37, 24.05.2014, BULU 36481, Ter.

Vicia villosa Roth subsp. villosa, 44, 14.05.2014, BULU 36071, Ter.

\section{FAGACEAE}

Fagus orientalis Lipsky, 26, 14.05.2015, BULU 38237 , Avr.-Sib., Fan.

Quercus cerris L., 68, 14.08.2015, BULU 39068A, Akd., Fan

Quercus frainetto Ten., 5, 11.05.2016, BULU 39688, Avr.Sib., Fan.

Quercus infectoria Oliv. subsp. infectoria, 25, 22.04.2014, BULU 35767, Avr.-Sib., Fan.

Quercus ithaburensis Decne subsp. macrolepis (Kotschy) Hedge \& Yalt., 32, 16.05.2017, BULU 41349, Fan.

Quercus petraea (Matt.) Liebl. subsp. iberica (Steven ex M. Bieb.) Krassiln., 6, 17.04.2014, BULU 35512, Fan.

Quercus pubescens Willd. subsp. pubescens, 19, 30.07.2017, BULU 41961, Fan.

Ouercus robur L. subsp. robur, 26, 25.06.2015, BULU 38409, Avr.-Sib., Fan.

Quercus trojana Webb subsp. trojana, 6, 17.04.2014, BULU 35511, D. Akd., Fan.

\section{GENTIANACEAE}

Centaurium erythraea subsp. turcicum (Velen.) Melderis, 10, 27.06.2014, BULU 37368, Hem.

\section{GERANIACEAE}

Erodium ciconium (L.) L' Her., 39, 16.05.2017, BULU 41247, Ter. 
Erodium cicutarium (L.) L' Her. subsp. cicutarium, 27, 14.05.2015, BULU 38245, Kozm., Ter.

Geranium lucidum L., 26, 22.04.2014, BULU 35706, Ter. Geranium purpureum Vill., 26, 14.05.2015, BULU 38206, Ter.

Geranium pusillum Burm.f., 25,22.04.2014, BULU 35762, Ter.

Geranium pyrenaicum Burm.f., 33, 16.04.2014, BULU 35390, Hem.

Geranium rotundifolium L., 34, 16.04.2014, BULU 35453, Ter.

\section{HYPERICACEAE}

Hypericum adenotrichum Spach, 29, 11.06.2014, BULU 37154, Hem., END., LC.

Hypericum aviculariifolium Jaub. \& Spach, 13, 27.06.2014, BULU 37351, D. Akd. Hem., END., LC.

Hypericum bourgaei (Sin: $\mathbf{H}$. aviculariifolium subsp. depilatum var. bourgaei (Boiss.) N.Robson), 46, 25.05.2014, BULU 36630, D. Akd., Hem., END., LC.

Hypericum calycinum L., 5, 21.07.2017, BULU 41848, İr.Tur., Hem.

Hypericum confertum choisy subsp. confertum, 29, 16.04.2014, BULU 35437, Hem., END., LC.

Hypericum montbretii Spach, 35, 23.06.2017, BULU 41540, Hem.

Hypericum olympicum L., 39, 10.07.2014, BULU 37613, D. Akd., Hem.

Hypericum orientale L., 34, 11.06.2014, BULU 37182, Hem.

Hypericum origanifolium Willd. var. origanifolium, 44, 22.05.2015, BULU 38284, Hem.

Hypericum perfoliatum L., 29, 24.09.2014, BULU 37997, Akd., Hem.

Hypericum perforatum L. subsp. perforatum, 9, 25.05.2014, BULU 36502, Hem.

50.JUGLANDACEAE

Juglans regia L., 5, 21.07.2017, BULU 41862, Fan. 51.LAMIACEAE

Ajuga chamaepitys (L.) Schreb subsp. chia (Schreb.) Arcang., 1, 22.05.2015, BULU 38346, Hem.

Ballota nigra L. subsp. anatolica P. H. Davis, 26, 25.06.2015, BULU 38397, İr.-Tur., Hem.

Clinopodium alpinum (L.) Kuntze (Sin: Acinos alpinum (L.) Moench), 9, 17.04.2014, BULU 35525, Hem.

Clinopodium suaveolens (Sm.) Kuntze (Sin: Acinos rotundifolius Pers.), 15, 11.06.2014, BULU 37235, Hem.

Clinopodium vulgare L. subsp. vulgare, 26, 16.06.2014, BULU 36266A, Avr.-Sib., Hem.

Lamium amplexicaule L. var. amplexicaule, 12, 16.04.2014, BULU 35488, Ter.

Lamium garganicum L. subsp. garganicum var. striatum (Sin: L. garganicum L. subsp. reniforme (Montbret \& Aucher ex Benter.) R.R.Mill.), 14, 19.04.2017, BULU 41189, Akd., Hem.

Lamium purpureum L. var. aznavourii Gand. ex Aznav., 23, 30.03.2013, BULU 40354, İr.-Tur., Ter., END., CR.

Lamium purpureum L. var. purpureum, 13, 19.04.2017, BULU 41193, Avr.-Sib., Ter.

Marrubium peregrinum L., 35, 23.06.2017, BULU 41607, Hem.

Marrubium vulgare L., 26, 22.04.2014, BULU 35698,
Akd., Hem.

Mentha longifolia (L.) L. subsp. thyphoides (Briq.) Harley (Sin: M. longifolia (L.) L. subsp thyphoides (Briq.) Harley var. typhoides (Briq.) Briq.), 39, 08.06.2017, BULU 41416, Hem.

Mentha pulegium L., 26, 13.07.2016, BULU 40224, Hem. Melissa officinalis L. subsp. officinalis, 21, 30.07.2017, BULU 42058, Avr.-Sib., Ter.

Micromeria myrtifolia Boiss. \& Hohen., 15, 11.06.2014, BULU 37238, Hem.

Nepeta italica L., 30, 11.06.2014, BULU 37167A, Akd., Hem.

Origanum vulgare L. subsp. gracile (K. Koch) Ietsw., 27, 23.06.2017, BULU 41640, İr.-Tur., Hem.

Origanum vulgare L. subsp. hirtum (Link) Ietsw., 26, 25.06.2015, BULU 38440, D. Akd., Hem.

Origanum vulgare L. subsp. viridulum (Martrin-Donos) Nyman (Sin: O. vulgare L. subsp. viride (Boiss.) Hayek), 10, 27.06.2014, BULU 37363, Hem.

Origanum sipyleum L., 19, 30.07.2017, BULU 41978, D. Akd., Hem., END., LC.

Prunella laciniata (L.) L., 34, 11.06.2014, BULU 37176 , Avr.-Sib., Hem.

Prunella vulgaris L., 26, 23.06.2016, BULU 40216, Avr.Sib., Hem.

Salvia forskahlei L., 10, 27.06.2014, BULU 37364, Avr.Sib., Hem.

Salvia sclarea L., 26, 23.06.2016, BULU 40214, Hem.

Salvia tomentosa Mill., 29, 11.06.2014, BULU 37151, Akd., Hem.

Salvia verbanaca L., 27, 14.05.2015, BULU 38256, Akd., Hem.

Salvia virgata Jacq., 21, 30.07.2017, BULU 42056, İr.Tur., Hem.

Salvia viridis L., 30, 11.06.2014, BULU 37168A, Akd., Ter.

Sideritis lanata L., 37, 24.05.2014, BULU 36477, D. Akd., Ter.

Sideritis montana L. subsp. montana, 31, 11.06.2014, BULU 37102, D. Akd., Ter.

Sideritis sipylea Boiss., 19, 30.07.2017, BULU 41977, D. Akd., Hem., END., EN.

Sideritis pisidica Boiss. \& Heldr., 27, 16.06.2014, BULU 36627, D. Akd., Hem., END., LC.

Stachys annua (L.) L. var. lycaonica R. Bhattacharjee, 1, 22.05.2015, BULU 38356, İr.-Tur., Ter.

Stachys byzantina K. Koch, 44, 24.05.2014, BULU 36433, Avr.-Sib., Hem.

Stachys cretica L. subsp. bulgarica Rech. f., 35, 23.06.2017, BULU 41581, D. Akd., Hem.

Stachys thirkei K. Koch, 13, 27.06.2014, BULU 37338, Hem.

Teucrium chamaedrys L. subsp. chamaedrys, 44, 09.07.2014, BULU 37540, Kam.

Teucrium orientale L. var. glabrescens Hausskn. ex Bornm., 35, 23.06.2017, BULU 41532, İr.-Tur., Hem.

Teucrium polium L. subsp. polium, 11, 25.05.2016, BULU 39705, Hem.

Thymus atticus Celak, 11, 25.05.2016, BULU 39714, Akd., Kam.

Thymus longicaulis C. Presl. subsp. chaubardii (Rchb.f.) 
Jalas, 1, 22.05.2015, BULU 38366, Akd., Kam.

Thymus longicaulis C. Presl subsp. longicaulis (Sin: T. longicaulis C. Presl subsp. longicaulis var. subisophyllus (Borbas) Jalas), 29, 11.06.2014, BULU 37147, Avr.-Sib., Kam.

Vitex agnus-castus L., 24, 25.05.2014, BULU 36673 B, Akd., Fan.

Ziziphora capitata L., 15, 11.06.2014, BULU 37237, Ter. Ziziphora taurica M. Bieb. subsp. taurica, 13, 27.06.2014, BULU 37264, Ter.

52.LINACEAE

Linum bienne Mill., 9, 16.05.2017, BULU 41405, Hem.

Linum corymbulosum Rchb., 31, 11.06.2014, BULU 37113, Akd., Ter.

Linum hirsutum L. subsp. anatolicum (Boiss.) Hayek var. platyphyllum P. H. Davis, 1, 22.05.2015, BULU 38344, Hem., END., EN.

Linum nodiflorum L., 1, 22.05.2015, BULU 38355, Akd., Ter.

Linum pamphylicum Boiss. \& Heldr. ex Planch subsp. olympicum G. Kaynak \& Ö. Yılmaz, 8, 25.05.2016, BULU 39763, Hem., END., CR.

53.LYTHRACEAE

Lythrum salicaria L., 26, 25.06.2015, BULU 38406, Avr.Sib., Hem.

\section{MALVACEAE}

Alcea biennis Winterl (Sin. A. pallida Waldst. \& Kit.), 36, 10.07.2014, BULU 37630, Hem.

Malva sylvestris L., 35, 10.06.2016, BULU 39962, Hem.

Tilia tomentosa Moench (Sin: T. argentea DC.), 26, 22.04.2014, BULU 35702, Fan.

\section{MORACEAE}

Ficus carica L. subsp. carica, 21, 30.07.2017, BULU 42067, Akd., Fan.

\section{OLEACEAE}

Fraxinus ornus L. subsp. ornus, 6, 19.04.2016, BULU 39626, Avr.-Sib., Fan.

Jasminum fruticans L., 44, 14.05.2014, BULU 36073, Akd., Fan.

Ligustrum vulgare L., 26, 22.04.2014, BULU 35737, Avr.Sib., Fan.

Phillyrea latifolia L., 32, 16.05.2017, BULU 41347, Akd., Fan.

\section{ONAGRACEAE}

Circaea lutetiana L., 26, 23.06.2016, BULU 40219, Hem. Epilobium hirsutum L., 26, 23.06.2016, BULU 40222, Hem.

Epilobium montanum L., 34, 11.06.2014, BULU 37175, Avr.-Sib., Hem.

Epilobium palustre L., 26, 25.06.2015, BULU 38384, Hem.

\section{OROBANCHACEAE}

Bellardia trixago L., 31, 11.06.2014, BULU 37111, Ter. Orobanche alba Stephan ex Willd. subsp. alba, 8, 25.05.2016, BULU 39727, Epi.

Orobanche fluginosa Reut. ex Jard,. 8, 16.05.2017, BULU 41372, Akd., Epi.

Orobanche lavandulacea Rchb., 26, 14.05.2015, BULU 38236, Akd., Epi.

Orobanche minor Sm., 37, 24.05.2014, BULU 36447, Epi. Parentucellia latifolia (L.) Caruel subsp. latifolia, 33,
16.04.2014, BULU 35388, Akd., Ter.

59.PAPAVERACEAE

Chelidonium majus L., 26, 22.04.2014, BULU 35676 , Avr.-Sib., Hem

Corydalis solida (L.) Clairv. subsp. solida, 26, 12.03.2015, BULU 38076, Kri.

Fumaria densiflora DC., 14, 19.04.2017, BULU 41198, Ter.

Fumaria officinalis L. subsp. officinalis, 34, 16.05.2017, BULU 41340A, Ter.

Fumaria vaillantii Loisel., 36, 23.06.2017, BULU 41441, Ter.

Papaver argemone L. subsp. argemone, 22, 16.05.2017, BULU 41263, Ter.

Papaver pilosum Sibter. \& Sm. subsp. strictum (Boiss. \& Balansa) N. Wendt ex Kadereit, 26, 16.06.2014, BULU 36251, Hem., END., VU.

Papaver rhoeas L., 11, 25.05.2016, BULU 39726, Ter. 60.PHYLLANTHACEAE

Andrachne telephioides L., 35, 30.07.2017, BULU 42032 , Hem.

\section{PLANTAGINACEAE}

Linaria corifolia Desf., 8, 19.04.2016, BULU 39620, İr. Tur., Hem., END., LC.

Linaria pelisseriana (L) Mill., 35, 23.06.2017, BULU 41538, Akd., Ter.

Linaria simplex DC., 36, 23.06.2017, BULU 41492, Akd., Ter.

Globularia trichosantha Fisch. \& C. A. Mey. subsp. trichosantha, 12, 22.04.2014, BULU 35783, İr.-Tur., Hem.

Gratiola officinalis L., 26, 23.06.2016, BULU 40217, Avr.Sib., Hem.

Plantago lanceolata L., 36, 23.06.2017, BULU 41510 Hem.

Plantago lagopus L., 35, 23.06.2017, BULU 41524, Akd., Ter.

Plantago scabra Moench, 40, 21.07.2017, BULU 41670 Ter.

Veronica arvensis L., 26, 14.05.2015, BULU 38213, Avr.Sib., Ter.

Veronica chamaedrys L., 30, 16.04.2014, BULU 35414, Avr.-Sib., Hem.

Veronica cymbalaria Bodard, 26, 22.04.2014, BULU 35731, Akd., Ter.

Veronica pectinata L. var. pectinata, 4, 26.03.2014, BULU 36055, Hem.

Veronica pectinata L. var. schzocalyx (Freyn \& Sint.) Bornm., 27, 14.05.2015, BULU 38238, Hem.

Veronica persica Poir., 23, 30.03.2013, BULU 40358, Ter. Veronica praecox All., 26, 14.05.2015, BULU 38182, Ter. 62. PLATANACEAE

Platanus orientalis L., 21, 30.07.2017, BULU 42062, Fan. 63. PLUMBAGINACEAE

Armeria cariensis Boiss. var. cariensis, 27, 14.05.2015, BULU 38258, D. Akd., Hem.

64.POLYGALACEAE

Polygala anatolica Boiss. \& Heldr., 11, 25.05.2016, BULU 39710, Hem.

Polygala pruinosa Boiss. subsp. pruinosa, 46, 25.05.2014, BULU 36607, Hem. 
Polygala supina Schreb. subsp. supina, 8, 25.05.2016, BULU 39729, Hem.

\section{POLYGONACEAE}

Polygonum arenastrum Boreau, 27, 23.06.2017, BULU 41646, Ter.

Polygonum lapathifolium L., 21, 30.07.2017, BULU 42044, Ter.

Polygonum persicaria L., 21, 30.07.2017, BULU 42050, Ter.

Rumex acetosella L., 37, 24.05.2014, BULU 36478, Hem. Rumex crispus L., 21, 30.07.2017, BULU 42045, Hem.

Rumex obtusifolius L. subsp. subalpinus (Schur) Celak, 27, 23.06.2017, BULU 41632, Hem.

Rumex tuberosus L. subsp. horizontalis (K. Koch) Rech. f., 27, 25.05.2014, BULU 36685, Hem.

Rumex tuberosus L. subsp. tuberosus, 39, 08.06.2017, BULU 41407, Hem.

\section{PRIMULACEAE}

Anagallis arvensis L. var. arvensis, 1, 22.05.2015, BULU 38347, Ter.

Anagallis arvensis L. var. caeurulea (L.) Gouan, 36, 23.06.2017, BULU 41507, Ter.

Lysimachia atropurpurea L., 9, 25.05.2014, BULU 36511, D. Akd., Hem.

Lysimachia verticillaris Spreng., 26, 23.06.2016, BULU 40218, İ.-Tur., Hem.

Primula acaulis (L.) L. subsp. acaulis (Sin: P. vulgaris Huds. subsp. sibthorpii (Hoffmanns.) N.W. Sm \& Forrest), 29, 16.04.2014, BULU 35445, Avr.-Sib., Kri.

\section{RANUNCULACEAE}

Anemone blanda Schott \& Kotschy, 11, 17.04.2015, BULU 38161, Kri.

Clematis vitalba L., 7, 21.07.2017, BULU 41846, Hem.

Clematis viticella L., 11, 17.04.2015, BULU 38362, Hem.

Nigella arvensis L. var. glauca Boiss., 36, 02.09.2014, BULU 37991, Ter.

Nigella sativa L., 44, 09.07.2014, BULU 37546, Ter.

Ranunculus constantinopolitanus (DC.) d'Urv., 29, 16.04.2014, BULU 35443, Hem.

Ranunculus damascenus Boiss. \& Gaill., 47, 11.06.2014, BULU 41333, İr.-Tur., Hem.

Ranunculus ficaria L. subsp. ficariiformis Rouy \& Foucaud, 24, 30.03.2013, BULU 40331, Hem.

Ranunculus gracilis E.D.Clarke, 46, 16.05.2017, BULU 41274, Hem.

Ranunculus neapolitanus Ten., 8, 17.04.2015, BULU 38179, Hem.

\section{RESEDACEAE}

Reseda lutea L. var. lutea, 35, 30.07.2017, BULU 41994, Hem.

69.RHAMNACEAE

Paliurus spina-christii P. Mill., 11, 30.07.2017, BULU 42075, Fan

\section{ROSACEAE}

Agrimonia eupatoria L. subsp. eupatoria, 35, 23.06.2017, BULU 41572, Hem.

Crataegus monogyna Jacq. var. monogyna, 26, 22.04.2014, BULU 35729, Fan.

Filipendula vulgaris Moench, 8, 25.05.2016, BULU 39753, Avr.-Sib., Hem.

Fragaria vesca L., 30, 16.04.2014, BULU 35405, Avr.-
Sib., Hem.

Geum urbanum L., 6, 19.04.2016, BULU 39628, Avr.-Sib., Hem.

Potentilla astranica Jacq. subsp. astranica, 45, 22.05.2015, BULU 38309, Avr.-Sib., Hem.

Potentilla buccoana Clem., 18, 19.04.2016, BULU 38681 A, İr.-Tur., Hem., END., VU.

Potentilla detommasii Ten., 26, 25.06.2015, BULU 38421, Hem.

Potentilla inclinata Vill., 6, 11.05.2016, BULU 38681B, Hem.

Prunus divaricata Ledeb. var. divaricata, 8, 17.04.2015, BULU 38181, Fan.

Pyracantha coccinea M.Roem., 35, 25.09.2017, BULU 42079, Avr.-Sib., Fan.

Pyrus eleagnifolia Pall. subsp. eleagnifolia, 26, 22.04.2014, BULU 35704, Fan.

Rosa canina L., 36, 23.06.2017, BULU 41435, Fan.

Rubus canescens DC. var. glabratus (Godr.) Davis \& Meikle, 9, 25.05.2014, BULU 36505, Avr.-Sib., Fan.

Sanguisorba minor L. subsp. lasiocarpa (Boiss. \& Hausskn.) Nordborg, 26, 22.04.2014, BULU 35727, Hem. 71.RUBIACEAE

Asperula arvensis L., 36, 23.06.2017, BULU 41513, Ter. Asperula involucrata Wahlenb., 6, 17.04.2014, BULU 35515, Avr.-Sib., Hem.

Asperula lilaciflora Boiss. subsp. phrygia (Bornm.) Schönb.-Tem., 13, 27.06.2014, BULU 37348, D. Akd., Hem., END., LC.

Crucianella angustifolia L., 35, 23.06.2017, BULU 41565, Akd., Ter.

Crucianella bithynica Boiss., 27, 11.06.2014, BULU 37251, D. Akd., Ter.

Crucianella latifolia L., 31, 11.06.2014, BULU 37119, Akd., Ter.

Cruciata pedemontana (Bellardi) Ehrend., 34, 16.05.2017, BULU 41332B, Ter.

Cruciata taurica (Pall. ex Willd.) Ehrend., 34, 17.04.2014, BULU 35647, İ.-Tur., Hem.

Galium album Mill. subsp. prusense (K. Koch.) Ehrend. \& Krendl, 16, 24.05.2014, BULU 36415, Hem.

Galium aparine L., 30, 16.04.2014, BULU 35399, Ter.

Galium divaricatum Pourr. ex Lam., 26, 23.06.2016, BULU 40198, Ter.

Galium fissurense Ehrend. \& Schönb.-Tem., 3, 10.06.2014, BULU 37087, İr.-Tur., Hem., END., LC.

Galium floribundum Sm. subsp. floribundum, 35, 23.06.2017, BULU 41534, Ter.

Galium murale (L.) All., 44, 22.05.2015, BULU 38292 , Akd., Ter.

Galium paschale Forssk., 35, 30.07.2017, BULU 42009, D. Akd., Hem.

Galium peplidifolium Boiss., 44, 14.05.2014, BULU 36083, D. Akd., Ter.

Galium setaceum Lam., 27, 16.06.2014, BULU 36265B, Ter.

Galium spurium L. subsp. spurium, 26, 22.04.2014, BULU 35688, Avr.-Sib., Ter.

Galium tricornutum Dandy, 8, 17.04.2014, BULU 38180, İr.-Tur., Ter.

Galium verticillatum Danthoine ex Lam., 44, 22.05.2015, 
BULU 38291, Akd., Ter.

Sherardia arvensis L., 30, 16.04.2014, BULU 35409, Akd.,

Ter.

\section{RUTACEAE}

Ruta buxbaumii Poir. (Sin: Haplophyllum buxbaumii (Poir.) G. Don), 7, 25.05.2014, BULU 36624, Hem.

\section{SALICACEAE}

Populus alba L. var. alba, 16, 30.07.2017, BULU 41988, Avr.-Sib., Fan.

Populus nigra L. subsp. nigra, 11, 25.05.2014, BULU 36538, Avr.-Sib., Fan.

Populus tremula L. subsp. tremula, 26, 16.06.2014, BULU 36263, Fan.

Salix alba L. subsp. alba, 13, 19.04.2017, BULU 41201, Avr.-Sib., Fan.

Salix caprea L., 7, 17.04.2014, BULU 35510, Avr.-Sib., Fan.

Salix $\times$ fragilis L., 23, 30.03.2013, BULU 40360, Avr.-Sib., Fan.

Salix viminalis L., 26, 23.06.2016, BULU 40204, Avr.Sib., Fan.

\section{SANTALACEAE}

Comandra umbellata (L.) Nutt. subsp. elegans (Roch. ex Sprengel) Piehl, 13, 22.04.2014, BULU 35796, Avr.-Sib., Hem.

Osyris alba L., 5, 23.06.2017, BULU 41660, Akd., Fan.

Thesium bergeri Zucc., 44, 24.05.2014, BULU 36434, D. Akd., Hem.

Thesium billardieri Boiss., 44, 09.07.2014, BULU 37542, İr.-Tur., Hem.

\section{SAPINDACEAE}

Acer campestre L. subsp. campestre, 26, 14.05.2015, BULU 38209, Avr.-Sib., Fan.

\section{SAXIFRAGACEAE}

Saxifraga adscendens L. subsp. adscendens, 26, 22.04.2014, BULU 35726, Hem.

Saxifraga sibirica L., 27, 07.04.2016, BULU 39516, Hem. Saxifraga tridactylites L., 23, 30.03.2013, BULU 40345, Akd., Ter.

\section{SCROPHULARIACEAE}

Scrophularia canina L. subsp. bicolor (Sm.) Greuter, 38, 10.07.2014, BULU 37614, D. Akd., Hem.

Verbascum basivelatum Hub.-Mor., 3, 10.06.2014, BULU 37090, İr.-Tur., Hem., END., NT.

Verbascum cheiranthifolium Boiss. var. asperulum (Boiss.) Murb., 35, 23.06.2017, BULU 41601, Hem.

Verbascum cheiranthifolium Boiss. var. cheiranthifolium, 28, 11.06.2014, BULU 37162A, Hem.

Verbascum flavidum (Boiss.) Freyn \& Bornm., 47, 11.06.2014, BULU 41334, Avr.-Sib., Hem.

Verbascum glomeratum Boiss., 15, 11.06.2014, BULU 37241, İr.-Tur., Hem.

Verbascum gnaphalodes M. Bieb., 2, 10.06.2014, BULU 37093, İr.-Tur., Hem.

Verbascum kastamunicum Murb., 45, 22.05.2015, BULU 38298, İr.-Tur., Hem., END., LC.

Verbascum luciliae (Boiss.) Kuntze, 27, 16.06.2014, BULU 36272, Hem., END., EN.

Verbascum lydium Boiss. var. lydium, 26, 25.06.2015, BULU 38388, Akd., Hem., END., LC.

Verbascum ovalifolium Donn ex Simas subsp. thracicum
(Velen.) Murb., 13, 27.06.2014, BULU 37337, Avr.-Sib., Hem.

Verbascum parviflorum Lam., 26, 16.06.2014, BULU 36271, D. Akd., Hem., END., LC.

Verbascum prusianum Boiss., 7, 21.07.2017, BULU 41847, Avr.-Sib., Hem., END., EN.

\section{SOLANACEAE}

Datura stramonium L., 27, 23.06.2017, BULU 41643, Hem.

Hyoscyamus niger L., 11, 25.05.2016, BULU 38171, Hem. Physalis alkekengi L., 21, 30.07.2017, BULU 42054, Ter. Solanum americanum Mill. (Sin: S. nigrum L. ), 26, 30.11.2012, BULU 40322, Hem.

\section{TAMARICACEAE}

Tamarix parviflora DC., 9, 16.05.2017, BULU 41364, Akd., Fan.

\section{URTICACEAE}

Parietaria judaica L., 26, 22.04.2014, BULU 35678, Hem. Urtica dioica L. subsp. dioica, 47, 23.06.2017, BULU 41571A, Avr.-Sib., Hem.

81. VERBANACEAE

Phyla canescens (Kunth) Greene, 17, 19.04.2016, BULU 39608, Hem

Verbana officinalis L. var. officinalis, 7, 21.07.2017, BULU 41826, Hem.

82.VIOLACEAE

Viola arvensis Murray, 47, 11.06.2014, BULU 41335, Ter. Viola canina L., 17, 19.04.2016, BULU 39600, Hem.

Viola odorata L., 27, 01.03.2016, BULU 39492, Hem.

\section{TARTIŞMA}

Çalışmanın sonucunda alandan 2011-2017 yılları arasında 47 farklı istasyondan yaklaşık 2100 bitki örneği toplanmıştır. Teşhisleri yapılan bitki örneklerinin 82 familyaya ait, 347 cins, 485 tür, 157 alt tür, 70 varyete ve 1 melez (Salix $\times$ fragilis) olmak üzere toplam 713 takson olduğu tespit edilmiştir. Taksonlardan 14'ü Pteridophyta, 699'u Spermatophyta bölümüne aittir. 8'i Pinophytina, 691’i Magnoliophytina alt bölümünde, 583’ü Magnoliopsida, 108’i Liliopsida sınıfında yer almaktadır.

Taksonların Raunkier'in hayat formlarına göre dağılımları ve oranları 59 (\% 8.3)'u Fanerofit, 328 (\% 46)'i Hemikriptofit, 78 (\% 10.9)'i Kriptofit, 231 (\% 32.4)'i Terofit, 13 (\%1.8)'ü Kamefit ve 4 (\% 0.6)'ü Epifit olarak bulunmuştur. Hemikriptofitlerin birinci sırada çıkmasının temel nedeni, dünyanın ılıman bölgelerinde yaygın olmaları, Akdeniz iklimine karakteristik olan Terofitlerin ikinci sırada bulunması alanının iklim yapisinin uygun olmasindan kaynaklanmaktadır.

Bitkilerin fitocoğrafik bölgelere göre dağılımları ve oranları Akdeniz elementi 149 (\%20.9), AvrupaSibirya elementi 89 (\%12.5), İran-Turan elementi 50 (\%7) ve çok bölgeli yada bölgesi bilinmeyenler 425 (\%59.6) şeklinde belirlenmiştir (Şekil 2). Çalışma alanı ve yakın çevrede yapılmış diğer çalışmalarda (Uludağ florası hariç) Akdeniz elementleri ilk sırada yer almaktadır. İkinci sırada Avr.-Sib., üçüncü sırada İr.- 
Tur. Elementleri bulunmaktadır. Akdeniz elementlerinin ilk sırada yer alması, alanın Akdeniz fitocoğrafik bölgesinde ve yarı kurak Akdeniz ikliminin görüldüğü bölgede bulunmasından kaynaklanmaktadır. Akdeniz elementleri P. brutia ormanı altları, maki çalılıkları ve meşelik içlerinde oldukça yaygindır. Avrupa-Sibirya elementlerine gölgelik ve nemli orman altlarında, yaygın olarak su kenarlarında ve vadi iç kısımlarında rastlanmaktadır. İran-Turan elementleri çoğunlukla taşlık kayalık açık alanlar, çalılık içleri, yol ve tarla kenarlarında yetişmektedir. Alandan tespit edilen 713 taksondan 57'si endemik olup endemizm oranı \%8.0'dir. Alanlar endemizm oranları bakımından karşılaştırıldığında Uludağ (Daşkın ve Kaynak, 2010a, b) ve Murat Dağı (Çırpıcı, 1989)'nda en yüksek olduğu, Büyükorhan, Harmancık (Bursa) ilçelerinde (Bağçıvan ve Daşkın, 2019), Gölcük (Dursunbey)'de (Dirmenci, 2006), Ulus Dağında (Güner ve Akçiçek, 2013) çalışma alanındakine yakın olduğu, Bursa Şehir florası (Günay Daşkın ve Kaynak, 2006) ve Katırlı Dağında (Erdoğan ve ark., 2011) ise daha düşük olduğu görülmektedir (Şekil 2).

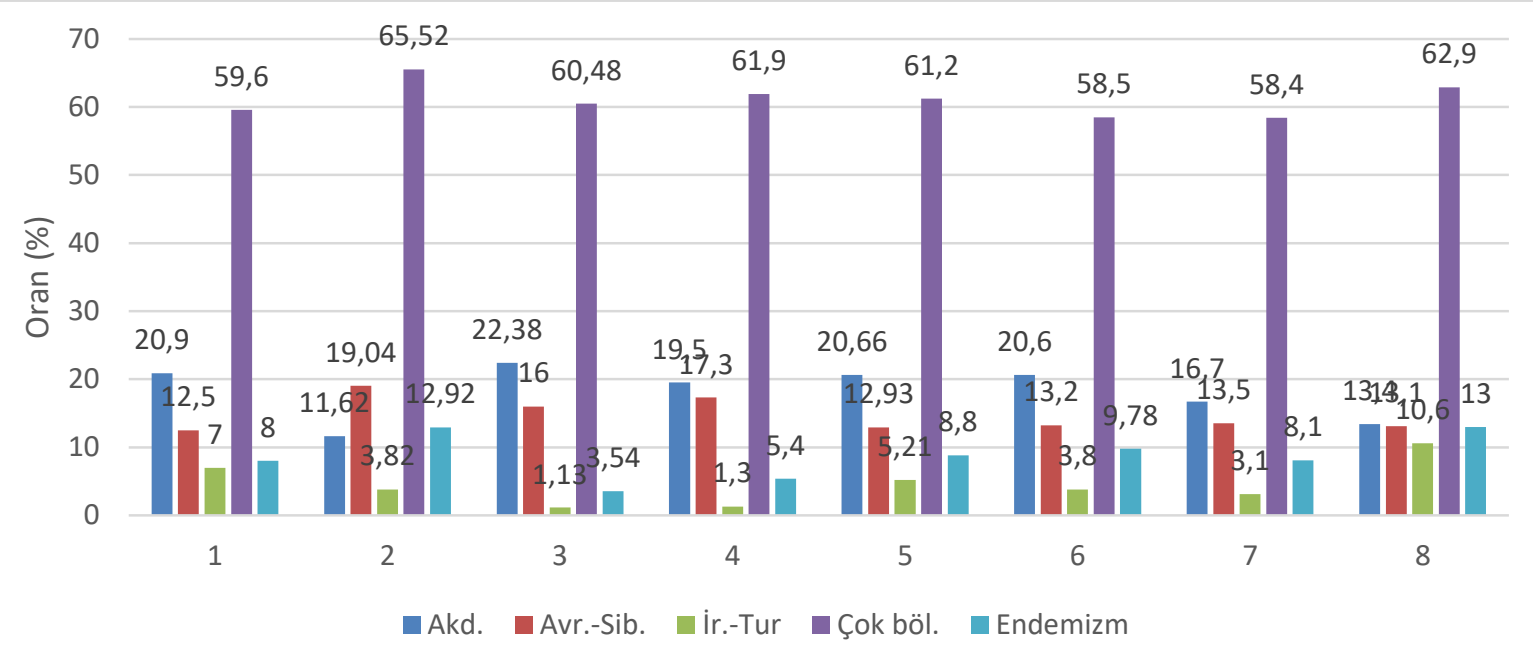

Şekil 2. Fitocoğrafik Bölge Elementleri ve Endemizm Oranlarının Yakın Bölgelerde Yapılmış Floristik Çalışmalar ile Karşılaştırılması

Figure 2. Comparision of Study Area and its Neigbour Areas in terms of Phytogeographical Region Elements and Endemism Rates

(1) Orhaneli (Bursa) florası; (2) Uludağ (Bursa) florası; (3) Bursa şehir florası; (4) Katırlı Dağı (Bursa florası; (5) Büyükorhan, Harmancık (Bursa) florası; (6) Gölcük (Dursunbey, Balıkesir) florası; (7) Ulus Dağı (Balikesir) florası; (8) Murat Dağı (Kütahya) florası

Araştırma alanında en fazla takson içeren familyalar ve içerdikleri takson sayıları Asteraceae (83), Fabaceae (64), Poaceae (50), Lamiaceae (45), Brassicaceae (42), Caryophyllaceae (41), Apiaceae (27),

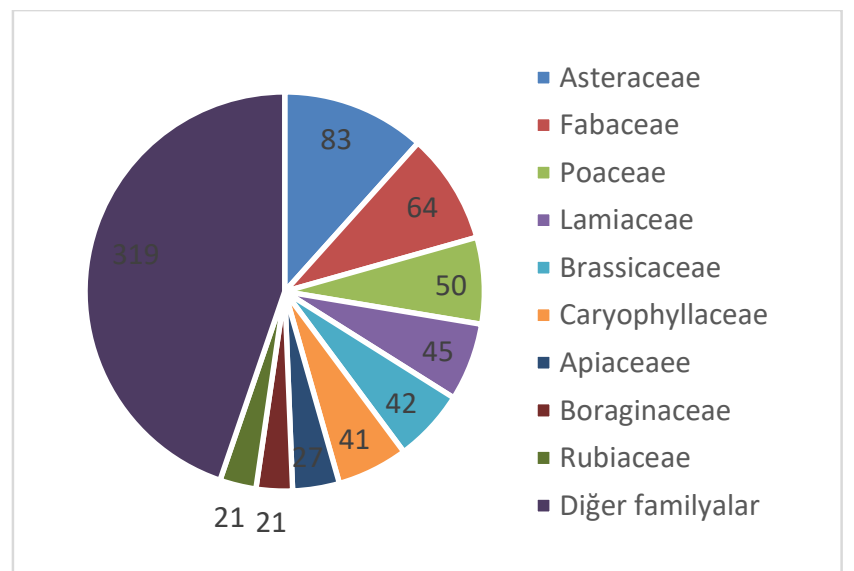

Şekil 3. Taksonların familyalara göre dağglımı Figure 3. Distribution of taxa according to the families
Boraginaceae ve Rubiaceae (21) şeklindedir (Şekil 3). En zengin cinsler Trifolium (18), Alyssum, Galium, Verbascum (12), Vicia, Hypericum (11), Euphorbia (9), Centaurea (8), Quercus (8) olarak bulunmuştur (Şekil $4)$.

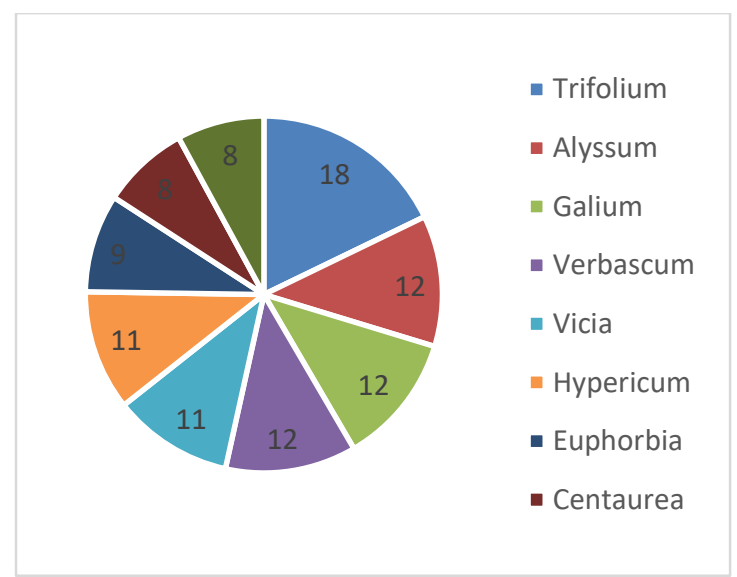

Şekil 4. En zengin cinsler ve içerdikleri takson sayıları Figure 4. The richest genera and the number of taxa they contain 
Çalışma alanında bitki çeşitliliğinin yüksek olduğu alanlar; yörede yetişen birçok bitki türü için sığınak oluşturan Sadağı Kanyonu içleri ve üstleri, Çeki-Sırıl yol ayrımı çevresi ve Nalınlar Çöreler arasıdır. Bu alanlarda bitki çeşitliliğini tehdit eden faktörler özellikle kanyondaki günü birlik ziyaretçi baskısı, kanyonun üst kısımlarında ise kısmen otlatma baskısı sayılabilir. Çeki-Sırıl yol ayrımı çevresi ve Nalınlar Çöreler arasında yol genişletme çalışmaları çeşitliliği tehdit eden en önemli faktör olarak gözlenmiştir. Bitki çeşitliliğin korunması amacı ile alınabilecek önlemler arasında Sadağı Kanyonundaki günü birlik ziyaretçilerin sayısının ve alan içindeki faaliyetlerinin denetlenmesi, otlatmaya ya hiç izin verilmemesi yada kontrollü bir şekilde yapılmasının sağlanması sayılabilir.

Alanda tespit edilen endemik taksonlardan 5'i Çok Tehlikede (CR), 6'sı Tehlikede (EN), 8'i Zarar Görebilir (VU), 6'sı Yakın zamanda tehlike altına Girebilir (NT), 30 'u Az endişe verici (LC) ve 2'si NE (Değerlendirilemedi) kategorisindedir (Ekim ve ark., 2000; Adıgüzel ve Reeves, 2002; Daşkın ve Kaynak, 2011; Çeçen, 2014). Sadağı Kanyonu içleri ve üst kesimleri, Çeki-Sırıl yol ayrımı çevresi, Harmancık yolu üzerindeki taşlık kayalık alanlar, Ağaçhisar Fadıl arası, Nalınlar, Deliballılar, Çöreler, Akçabük çevresi, Çınarcık Barajı üstleri endemik taksonların yetiştiği alanlardır. $\mathrm{Bu}$ alanların çoğunda ana kayanın serpantin olması ve son zamanlarda bölgeden yeni tür olarak tanımlanan taksonların da söz konusu alanlarda yayılış göstermesi dikkat çekicidir. Alanında yetişen endemiklerden Papaver pilosum ssp. strictum, Elaeosticta aurea, Centaurea wiedemanniana, Anthemis xylopoda, Centaurea ertugruliana, Verbascum luciliae, V. kastamunicum'un yayılışları oldukça lokal olup populasyonları azdır. Diğer yandan Crocus flavus ssp. dissectus, Alyssum dudleyi, Aubrieta olympica, Dianthus goekayi, Linum pamphylicum ssp. olympicum'un alandaki populasyonları nispeten daha iyi durumdadir.

$\mathrm{Bu}$ çalışma ile Bursa ili Orhaneli ilçesinde 713 vasküler bitki taksonunun yayılış gösterdiği saptanmış olup çalışmamızın ileride yörede yetişen bitkiler üzerinde araştırmalar yapacak bilim insanlarına katkı sağlayacağı kanaatindeyiz.

\section{TEŞEKKÜR}

$\mathrm{Bu}$ çalışma ilk yazarın doktora tezinin bir kısmını oluşturmakta olup çalışmayı maddi olarak destekleyen Bursa Uludağ Üniversitesi Bilimsel Araştırma Projeleri Birimine (Proje no: UAP (F) 2011/73) teşekkür ederiz.

\section{Çıkar Çatışması Beyanı}

Makale yazarları aralarında herhangi bir çıkar çatışması olmadığını beyan ederler.

\section{Araştırmacıların Katkı Oranı Beyan Özeti}

Yazarlar makaleye eşit oranda katkı sağlamış olduklarını beyan ederler.

\section{KAYNAKLAR}

Adıgüzel N, Reeves R D 2002. A new nickelaccumulating species of Alyssum (Cruciferae) from Western Turkey, Edinburgh Journal of Botany 59 (2): $215-219$.

Akman Y 1999. İklim ve Biyoiklim. Kariyer Matbaacilık, Ankara, 350 sy.

Anonim 1976-2006. Bursa Merkez istasyonuna Ait İklimsel Veriler, TC. Cevre ve Orman Bakanlığg, Devlet Meteoroloji İşleri Genel Müdürlüğü, Ankara.

Anonim 2010. Bursa İli Maden ve Enerji Kaynakları. T.C. Maden Tetkik Arama ve Genel Müdürlüğü Raporu, Ankara.

Anonim 2012. Orhaneli, Büyükorhan, Harmancık İlçeleri Genel Bilgiler. Bursa Eskişehir Bilecik Kalkınma Ajansı (BEBKA) Raporu, Bursa.

Anonim 2013-2016. Bursa ili Orhaneli İlçesine Ait İklimsel Veriler. TC. Çevre ve Orman Bakanllğ 1 , Devlet Meteoroloji İşleri Genel Müdürlügü, Bursa Meteoroloji İl Müdürlüğü, Bursa.

Avcı M 2005. Çeşitlilik ve Endemizm Açısından Türkiye'nin Bitki Örtüsü. Coğrafya Derg 13: 27-55.

Bağçıvan G, Daşkın R 2019. Büyükorhan ve Harmancık İlçelerinin （Bursa/Türkiye） Florası. Biological Diversity and Conservation 12(2):126140.

Çeçen Ö 2014. Çakırdağı'nın (Karaman) Florası. Dumlupınar Üniversitesi Fen Bilimleri Enstitüsü Biyoloji Ana Bilim Dalı, Doktora Tezi, 153 sy.

Çrpıcı A 1989. Murat Dağ DOĞA TU Botanik Derg 13 (2): 157-222.

Daşkın R, Kaynak G 2010 a. Vascular flora of the Uludag Mt (Bursa, Turkey)I. Phytologia Balcanica 16(3): 369-384.

Dasskın R, Kaynak G 2010 b. Vascular flora of the Uludag Mt (Bursa, Turkey)II. Phytologia Balcanica 16(3): 385-411.

Daşkın R, Kaynak G 2011. Conservation status of five endemic species distributed in Northwest Turkey. Phytologia Balcanica 17(2): 213-219.

Davis PH 1965-1985. Flora of Turkey and the East Aegean Islands, Volume 1-9, Edinburgh Univ. Press, Edinburgh.

Davis, PH, Mill RR, Tan K 1988. Flora of Turkey and the East Aegean Islands, Volume 10, Edinburgh Univ. Press, Edinburgh.

Dirmenci T 2006. Gölcük (Dursunbey, Balıkesir) Florası Üzerine Bir Çalışma, Ot Sistematik Botanik Dergisi 13(1): 97-124.

Ekim T, Koyuncu M, Vural M, Duman H, Aytaç Z, Adıgüzel N 2000. Türkiye Bitkileri Kırmızı Kitabı (Eğreltiler ve Tohumlu Bitkiler), Türkiye Doğa 
Koruma Birliği, Barışcan Ofset, Ankara.

Erdoğan E, Kaynak G, Daşkın R, Yılmaz Ö 2011. The Vascular Flora of Katırl Mountain (Bursa, Turkey). Biological Diversity and Conservation 4(1):148-170.

Günay Daşkın R, Kaynak G 2006. Bursa Şehir Florası. Ot Sistematik Botanik Derg 13(1): 125-158.

Güner A, Özhatay N, Ekim T, Başer KHC 2000. Flora of Turkey and the East Agean Islands, Volume 11 (Supplement 2), Edinburgh Univ. Press, Edinburgh.

Güner A, Aslan S, Ekim T, Vural M, Babaç MT 2012. Türkiye Bitkileri Listesi (Damarlı Bitkiler), Nezahat Gökyiğit Botanik Bahçesi Yayınları Flora Dizisi 1, İstanbul, $1290 \mathrm{~s}$.
Güner Ö, Akçiçek E 2013. Ulus Dağı'nın Florası (Balıkesir/Türkiye). Biological Diversity and Conservation 6(1): 101-113.

Malyer H, Daşkın R, Bağçıvan G 2016. Sadağ Kanyonu Tabiat Parkı Biyolojik Çeşitlilik Kitabı, Flora ve Vejetasyon, Orman ve Su İşleri Bakanlığı, II. Bölge Müdürlügü Bursa Şube Müdürlüğü, Seçil Ofset, Bursa, s. 31-201.

Raunkiaer C 1934. The Life Forms of Plants and Statistical Plant Geography. Oxford University Press, London.

Seçmen Ö, Leblebici E 2008. Türkiye Sulak Alan Bitkileri ve Bitki Örtüsü. Ege Üniversitesi Yayınları, Fen Fakültesi, Yayın No: 158 (2.Baskı), İzmir. 